\title{
Pacific
}

Journal of

Mathematics

\section{GENERALIZED SPLINES ON ARBITRARY GRAPHS}

Simcha Gilbert, Julianna Tymoczko and Shira Viel 


\title{
GENERALIZED SPLINES ON ARBITRARY GRAPHS
}

\author{
Simcha Gilbert, Julianna TymoczKo AND ShIRA Viel
}

Let $G$ be a graph whose edges are labeled by ideals of a commutative ring. We introduce a generalized spline, which is a vertex labeling of $G$ by elements of the ring so that the difference between the labels of any two adjacent vertices lies in the corresponding edge ideal. Generalized splines arise naturally in combinatorics (algebraic splines of Billera and others) and in algebraic topology (certain equivariant cohomology rings, described by Goresky, Kottwitz, and MacPherson, among others). The central question of this paper asks when an arbitrary edge-labeled graph has nontrivial generalized splines. The answer is "always", and we prove the stronger result that the module of generalized splines contains a free submodule whose rank is the number of vertices in $G$. We describe the module of generalized splines when $G$ is a tree, and give several ways to describe the ring of generalized splines as an intersection of generalized splines for simpler subgraphs of $G$. We also present a new tool which we call the GKM matrix, an analogue of the incidence matrix of a graph, and end with open questions.

\section{Introduction}

The goal of this paper is to generalize and extend combinatorial constructions that have become increasingly important in many areas of algebraic geometry and topology, as well as to establish a firm combinatorial footing for these constructions. Given a commutative ring $R$ with identity, an arbitrary graph $G=(V, E)$, and a function $\alpha: E \rightarrow$ ideals $I \subseteq R$, we will define a ring of generalized splines. This paper

(1) proves foundational results about generalized splines;

(2) completely analyzes the ring of generalized splines for trees and shows families of generalized splines for arbitrary cycles;

We are very grateful for the contributions of Arielle McCoy and Tia Pilaroscia, and for helpful conversations with Matthias Franz, Ruth Haas, and Hal Schenck. We are indebted to an anonymous referee for very useful comments.

This work was partially supported by NSF grant DMS-0611020 through the Center for Women in Mathematics at Smith College. Tymoczko was partially supported by a Sloan Fellowship and by NSF grants DMS-1101170 and DMS-0801554.

MSC2010: 05C78, 05E15, 55N25.

Keywords: splines, GKM theory, equivariant cohomology, algebraic graph theory. 


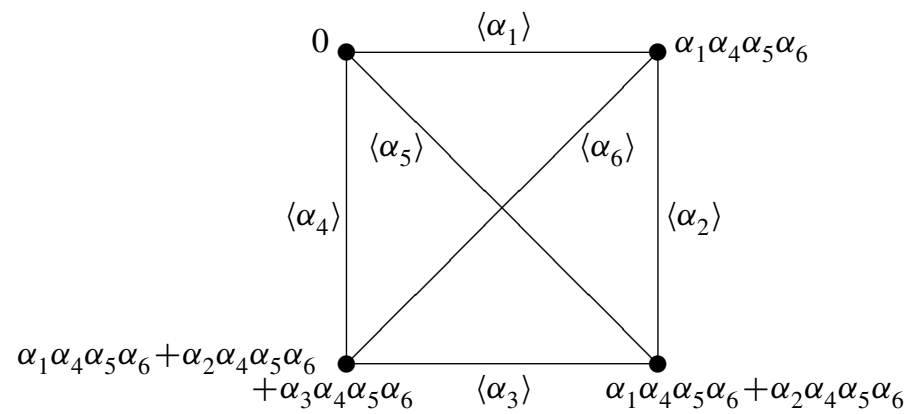

Figure 1. Example of a generalized spline on $K_{4}$.

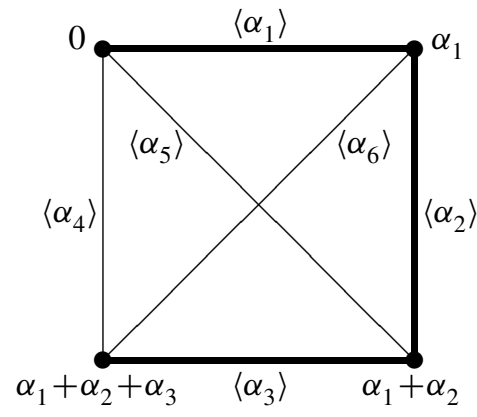

(a) Spline on subgraph $\left(P_{4},\left.\alpha\right|_{P_{4}}\right)$.

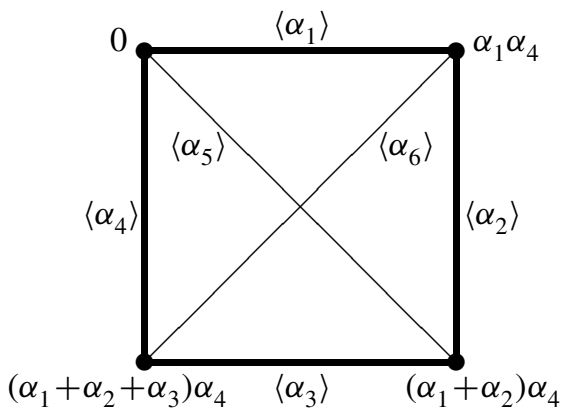

(b) Spline on subgraph $\left(C_{4},\left.\alpha\right|_{C_{4}}\right)$.

Figure 2. Nonexamples of generalized splines on $K_{4}$.

(3) produces an $R$-submodule within the ring of generalized splines that has rank $|V|$, as long as $R$ is an integral domain; and

(4) shows that the study of generalized splines for arbitrary graphs can be reduced to the case of different subgraphs, especially cycles or trees.

Generalized splines as we define them are a subring of a product of copies of $R$ :

Definition 1.1. The ring of generalized splines $R_{G}$ of the pair $(G, \alpha)$ is defined by

$$
R_{G}=\left\{\boldsymbol{p} \in R^{|V|}: \text { for each edge } e=u v, \text { the difference } \boldsymbol{p}_{u}-\boldsymbol{p}_{v} \in \alpha(e)\right\} .
$$

Figures 1 and 2 display examples and nonexamples of elements of $R_{K_{4}}$ in the case when each ideal $\alpha(e)$ is generated by a single ring element (given inside $\langle\cdot\rangle$ ). The vertices are labeled with elements of $R_{K_{4}}$ and the collection of vertex labels in Figure 1 is a generalized spline. Note that Figures 2(a) and 2(b) are not generalized splines on $K_{4}$ but are generalized splines on the subgraphs in bold. These examples hold for any ring $R$ and any choice of elements $\alpha_{1}, \alpha_{2}, \ldots, \alpha_{6} \in R$ to generate the ideals $\alpha(e)$. 
The name generalized spline comes from one of the important constructions that we extend. Historically, engineers modeled complicated objects like ships or cars by identifying important points of the vehicle and then attaching thin strips of wood (called splines) at those points to approximate the entire hull.

Mathematically, a spline is a collection of polynomials on the faces of a polyhedral complex that agree (modulo a power of a linear form) on the intersections of two faces. We refer to this classical tradition as the analytic approach to splines; it studies the vector space $C_{k}^{r}(D)$, where $D$ is a simplicial complex, $r$ is the order of smoothness to which the polynomials agree over faces, and $k$ is the maximal degree of a polynomial supported on a maximal face. Splines are used in approximation theory and numerical analysis, with applications in data interpolation, to create smooth curves in computer graphics, to find numerical solutions to partial differential equations, and for other applications [Bartels 1984; Chui and Lai 1985; 1990].

In the analytic tradition, mathematicians seek individual splines satisfying particular properties as well as characterizations of the space of splines associated to a given object - for instance, the dimension [Alfeld 1986; 1987; Alfeld and Schumaker 1987; 1990; Chui and He 1989; Gmelig Meyling and Pfluger 1985; Schumaker 1979; 1984a; 1984b] or basis [Alfeld et al. 1987a; 1987b; Morgan and Scott 1975; Schumaker 1988] for a space of splines. Alfeld and Schumaker's work is both representative and epitomic: a seminal result of theirs proved a bound on the dimension of $C_{k}^{r}(D)$ when $D$ is a planar simplicial complex and $k \geq 3 r+1$ [1987].

Billera [1988] pioneered the study of what some call algebraic splines, introducing methods from homological and commutative algebra to prove a conjecture of Strang on the dimension of $C_{k}^{1}(D)$ when $D$ is a generic planar simplicial complex. In the abstract algebraic setting, mathematicians generalize the class of geometric objects associated to splines (e.g., Schumaker [1984b], Billera and Rose [1991], and McDonald and Schenck [2009] study piecewise polynomials on a polyhedral complex rather than just a simplicial complex) and study algebraic invariants of modules other than dimension or bases (e.g., the more fundamental question of freeness [Haas 1991; Billera and Rose 1992; Yuzvinsky 1992; Dalbec and Schenck 2001; DiPasquale 2012], or more algebraically involved questions like computing coefficients of the Hilbert polynomial [Billera and Rose 1991; Schenck and Stillman 1997; Schenck 1997; McDonald 2007; McDonald and Schenck 2009], identifying the syzygy module of the span of the edge ideals [Schumaker 1979; Rose 1995; 2004], or analyzing algebraic varieties associated to the piecewise polynomials [Wang 2000; Zhu and Wang 2005; 2011]). Billera and Rose [1991] introduced a description of splines in terms of the dual graph of the polyhedral complex that is equivalent to the piecewise-polynomial definition for so-called hereditary complexes. Many others used Billera and Rose's approach in later research [McDonald and Schenck 2009; Rose 1995; 2004], and it is our starting point. 
In what we might call the topological approach to splines, geometers and topologists recently and independently rediscovered splines as equivariant cohomology rings of toric and other algebraic varieties (though they rarely use the name "splines") [Brion 1996; Payne 2006; Bahri et al. 2009; Schenck 2012]. Goresky, Kottwitz, and MacPherson developed a combinatorial construction of equivariant cohomology called GKM theory [Goresky et al. 1998], which can be used for any algebraic variety $X$ with an appropriate torus action. Unknowingly, they described precisely the dual-graph construction of splines: GKM theory builds a graph $G_{X}$ whose vertices are the $T$-fixed points of $X$ and whose edges are the one-dimensional orbits of $X$. Each edge of this graph is associated to a principle ideal $\left\langle\alpha_{e}\right\rangle$ in a polynomial ring, coming from the weight $\alpha_{e}$ of the torus action on the one-dimensional torus orbits in $X$. The GKM ring associated to the pair $\left(G_{X}, \alpha\right)$ agrees with what we call the ring of generalized splines for $\left(G_{X}, \alpha\right)$. The main theorem of GKM theory asserts that under appropriate conditions, this GKM ring is in fact isomorphic to the equivariant cohomology ring $H_{T}^{*}(X ; \mathbb{C})$. (Their work relies on earlier work of many others, including a much more general result of Chang and Skjelbred [1974] that points to one way to extend this work topologically to cases in which the ideals $\left\langle\alpha_{e}\right\rangle$ are no longer principal.) We omit details of the topological background here because there are several excellent surveys [Knutson and Tao 2003; Tymoczko 2005; Holm 2008]. However, GKM theory is a powerful tool in Schubert calculus [Goldin and Tolman 2009; Knutson and Tao 2003], symplectic geometry [Goldin and Tolman 2009; Guillemin et al. 2006; Harada et al. 2005], representation theory [Fiebig 2011], and other fields. (In some of these applications, the ring structure of splines is more important than the module structure.)

We note that the most powerful results in each of these approaches are not replicable using other approaches. For instance, Mourrain and Villamizar [2013] recently used the algebraic approach to try to re-prove Alfeld and Schumaker's results, but could not attain their bound.

Our definition of generalized splines allows us to do several things that weren't possible from the algebraic or geometric perspectives:

- We give a lower bound for one of the central questions of classical splines. Corollary 5.2 proves that every collection of generalized splines over an integral domain has a free submodule of rank $|V|$, producing a lower bound for the dimension of the ring of splines $R_{G}$ whenever $R_{G}$ is a free module over $R$. This significantly generalizes work of Guillemin and Zara in the GKM context [2003, Theorem 2.1].

- We streamline earlier combinatorial constructions of splines. Our construction isolates and highlights the algebraic structures used in previous work on splines. In our language, algebraic splines assume that the ideals $\alpha(e)$ are principal and that the generators for the ideals $\alpha(e)$ satisfy some coprimality conditions. A classical 
condition like "piecewise polynomials meet with order $r$ smoothness at an edge $e$ " corresponds to using the edge ideal $\alpha(e)^{r+1}$ instead of $\alpha(e)$.

From the geometric point of view, we owe much to a series of papers by Guillemin and Zara [2001; 2003] whose goal is to construct geometric properties of GKM manifolds from a strictly combinatorial viewpoint. Yet their combinatorial model imposes more restrictions than the classical definition of splines - conditions that are natural (and necessary!) for any geometric application.

- We expand the family of objects on which splines are defined to arbitrary graphs. Our work shows that graphs that have no reasonable geometric interpretation nonetheless are central to the analysis of splines. Theorem 6.1 decomposes the ring of splines for a graph $G$ in terms of the splines for subgraphs of $G$; Corollary 6.2 specializes Theorem 6.1 to spanning trees, whose splines are completely described in Theorem 4.1; and Theorem 6.3 decomposes the ring of splines for $G$ in terms of a particular collection of subcycles and subtrees of $G$. Cycles play a similarly key role in Rose's description of the syzygies of spline ideals [1995; 2004] (see also [Schumaker 1979]). Yet neither trees nor cycles are geometrically meaningful from a GKM perspective. (See [Handschy et al. 2014] and [Bowden et al. 2015] for a deeper investigation of generalized splines on cycles.)

- We expand the family of rings on which splines are defined. This gives a convenient language to describe simultaneously the GKM constructions for equivariant cohomology and equivariant $K$-theory. Moreover, generalized splines over integers have interesting connections to elementary number theory [Handschy et al. 2014].

- We provide the natural language for further generalizations of splines. Our construction of generalized splines extends even more: label each vertex of the graph $G$ by a (possibly distinct) $R$-module $M_{v}$ and label each edge by a module $M_{e}$ together with homomorphisms $M_{v} \rightarrow M_{e}$ for each vertex $v$ incident to the edge $e$. Geometrically, this corresponds to Braden and MacPherson's construction of equivariant intersection homology [2001], also used by Fiebig in representation-theoretic contexts [2011]. We discuss this and other open questions in Section 7.

The rest of this paper is structured as follows. Section 2 establishes essential results for generalized splines that were first shown in special cases like equivariant cohomology and algebraic splines. We highlight Theorem 2.12 and Corollary 2.13, which generalize and strengthen Rose's result [1995] that for certain polyhedral complexes, the syzygies $B$ of the spline ideal are a direct summand of the splines $R_{G} \cong R \oplus B$. Corollary 2.13 uses this in Rose's special case to show that the syzygies of the ideal generated by the image of $\alpha$ are isomorphic as a module to the collection of generalized splines whose restriction to a particular fixed point is zero. This relates the algebraically natural question of finding syzygies of splines to the question of finding a particular, geometrically natural kind of basis for the 
module of splines. Section 3 describes a tool analogous to the incidence matrix of a graph that we call a GKM matrix. Section 4 completely characterizes the generalized splines for trees in terms of a minimal set of free generators for the ring of generalized splines.

One of our central questions is, when does an edge-labeled graph have nontrivial generalized splines? The answer (essentially always, as in Theorem 5.1) is actually more refined. Corollary 5.2 explicitly constructs a free $R$-submodule of the generalized splines on $G$ of rank $|V|$. When $R$ is an integral domain and the generalized splines form a free $R$-module (as is the case for GKM theory), we conclude that the rank of the $R$-module of generalized splines is at least $|V|$.

Section 5 uses analogues of a shelling order (in combinatorics) or a "flow-up basis" (in geometry) to identify $R$-submodules of the generalized splines. Section 6 characterizes generalized splines differently: in terms of the intersections of the generalized splines formed by various subgraphs. This allows us to reframe the definition of generalized splines as an intersection of very simple graphs (Theorem 6.1) and to reduce the number of intersections needed by using certain spanning trees (Corollary 6.2). Finally, Theorem 6.3 analyzes the GKM matrix directly to decompose the ring of generalized splines on $G$ as an intersection of the generalized splines for particular subcycles of $G$.

\section{Definitions and foundational results}

In this section, we formalize a collection of definitions which were stated implicitly in the introduction. We then give foundational results describing the structure of the ring of generalized splines, including key methods to construct the ring and to build new generalized splines from existing ones.

We begin with a quick overview of our notational conventions.

- $G$ : a graph, defined as a set of vertices $V$ and edges $E$. Assumed throughout to be finite with no multiple edges between vertices.

- $R$ : a commutative ring with identity 1 .

- I: the set of ideals in $R$.

- $\alpha$ : an edge-labeling function on $G$ that assigns a nonzero element of $\mathcal{I}$ to each edge in $E$. See Definition 2.1.

- $(G, \alpha)$ : an edge-labeled graph.

- $\alpha\left(e_{i, j}\right)=\alpha\left(v_{i} v_{j}\right)=I_{e_{i, j}}$ : the image of the edge $e_{i, j}=v_{i} v_{j}$ under the map $\alpha$.

- $\alpha_{i, j}$ : an arbitrary element of the ideal $\alpha\left(e_{i, j}\right)$. When $\alpha\left(e_{i, j}\right)$ is principal, $\alpha_{i, j}$ often denotes the generator.

- $R_{G}$ : the ring of generalized splines on $(G, \alpha)$. See Definition 2.3. 
- $\boldsymbol{p}$ : a generalized spline; $\boldsymbol{p}=\left(\boldsymbol{p}_{v_{1}}, \boldsymbol{p}_{v_{2}}, \ldots, \boldsymbol{p}_{v_{|V|}}\right)$ denotes an element of $\bigoplus_{v \in V} R$. See Definition 2.3.

- $\boldsymbol{p}_{v}$ : the coordinate of $\boldsymbol{p}$ corresponding to vertex $v \in V$. An element of $R$.

- $M_{G}$ : the (possibly extended) GKM matrix for the graph G. See Definition 3.1.

The first definition describes the combinatorial setup of our work: a graph whose edges are labeled by ideals in a ring $R$. The ring $R$ is always assumed to be a commutative ring with identity, though in later sections we occasionally add more conditions.

Definition 2.1. Let $G=(V, E)$ be a graph and let $R$ be a commutative ring with identity. An edge-labeling function is a map $\alpha: E \rightarrow\{$ ideals $I \subseteq R\}$ from the set of edges of $G$ to the set of nonzero ideals in $R$. An edge-labeled graph is a pair $(G, \alpha)$ of a graph $G$ together with an edge-labeling function of $E$. We often refer to the set of ideals in $R$ as $\mathcal{I}$.

We now precisely define the compatibility condition that we use on the edges.

Definition 2.2. Let $G=(V, \alpha)$ be an edge-labeled graph. An element $\boldsymbol{p} \in \bigoplus_{v \in V} R$ satisfies the GKM condition at an edge $e=u v$ if $\boldsymbol{p}_{u}-\boldsymbol{p}_{v} \in \alpha(e)$.

In GKM theory and in the theory of algebraic splines, the ring $R$ is a polynomial ring in $n$ variables. The ideal $\alpha(e)$ is the principal ideal generated by a linear form in GKM theory, and by a power of a linear form in the theory of algebraic splines.

We build the ring of generalized splines by imposing the GKM condition at every edge in the graph.

Definition 2.3. Let $(G, \alpha)$ be an edge-labeled graph. The ring of generalized splines is

$R_{G, \alpha}=\left\{\boldsymbol{p} \in \bigoplus_{v \in V} R\right.$ such that $\boldsymbol{p}$ satisfies the GKM condition at each edge $\left.e \in E\right\}$.

Each element of $R_{G, \alpha}$ is called a generalized spline. When there is no risk of confusion, we write $R_{G}$.

We first confirm that in fact $R_{G}$ is a ring.

Proposition 2.4. $R_{G}$ is a ring with unit $\mathbf{1}$ defined by $\mathbf{1}_{v}=1$ for each vertex $v \in V$.

Proof. By definition $R_{G}$ is a subset of the product ring $\bigoplus_{v \in V} R$ so we need only confirm that the identity is in $R_{G}$ and that $R_{G}$ is closed under addition and multiplication. The operations are componentwise addition and multiplication since $R_{G}$ is in $\bigoplus_{v \in V} R$. The identity in $\bigoplus_{v \in V} R$ is the generalized spline $\mathbf{1}$ defined by $\mathbf{1}_{v}=1$ for each vertex $v \in V$. This satisfies the GKM condition at each edge because for 


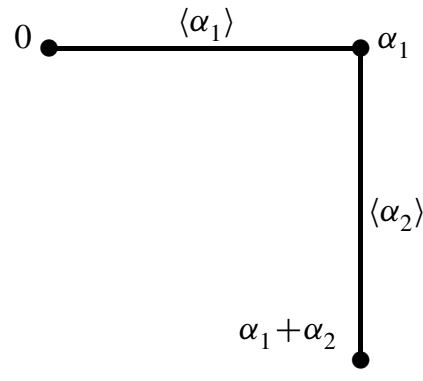

(a) $\left.\boldsymbol{p}\right|_{P_{3}} \in R_{P_{3}}$.

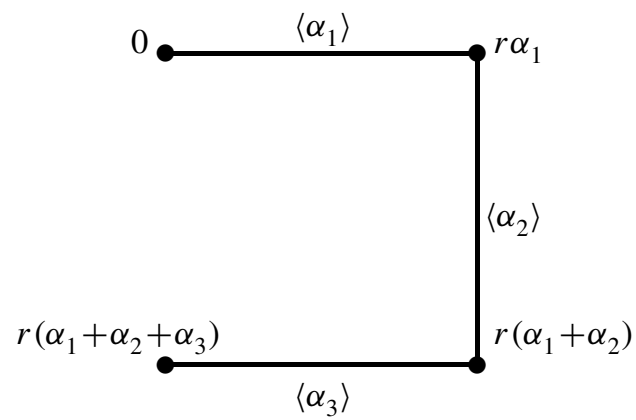

(b) $r \boldsymbol{p} \in R_{P_{4}}$.

Figure 3. New generalized splines from old.

every edge $e=u v$ we have $\mathbf{1}_{u}-\mathbf{1}_{v}=0$ and 0 is in each ideal $\alpha(e)$. The set $R_{G}$ is closed under addition because if $\boldsymbol{p}, \boldsymbol{q} \in R_{G}$ then for each edge $e=u v$ we have

$$
(\boldsymbol{p}+\boldsymbol{q})_{u}-(\boldsymbol{p}+\boldsymbol{q})_{v}=\left(\boldsymbol{p}_{u}+\boldsymbol{q}_{u}\right)-\left(\boldsymbol{p}_{v}+\boldsymbol{q}_{v}\right)=\left(\boldsymbol{p}_{u}-\boldsymbol{p}_{v}\right)+\left(\boldsymbol{q}_{u}-\boldsymbol{q}_{v}\right),
$$

which is in $\alpha(e)$ by the GKM condition. Similarly, the set $R_{G}$ is closed under multiplication because if $\boldsymbol{p}, \boldsymbol{q} \in R_{G}$ then for each edge $e=u v$ we have

$$
\begin{aligned}
(\boldsymbol{p q})_{u}-(\boldsymbol{p q})_{v} & =\left(\boldsymbol{p}_{u} \boldsymbol{q}_{u}\right)-\left(\boldsymbol{p}_{v} \boldsymbol{q}_{v}\right) \\
& =\left(\boldsymbol{p}_{u} \boldsymbol{q}_{u}-\boldsymbol{p}_{v} \boldsymbol{q}_{u}\right)+\left(\boldsymbol{p}_{v} \boldsymbol{q}_{u}-\boldsymbol{p}_{v} \boldsymbol{q}_{v}\right) \\
& =\boldsymbol{q}_{u}\left(\boldsymbol{p}_{u}-\boldsymbol{p}_{v}\right)+\boldsymbol{p}_{v}\left(\boldsymbol{q}_{u}-\boldsymbol{q}_{v}\right),
\end{aligned}
$$

which is in $\alpha(e)$ by the GKM condition.

The generalized splines $R_{G}$ also form an $R$-module: multiplication by $r$ corresponds to scaling each polynomial in the spline $\boldsymbol{p}$ or equivalently to multiplication by $r \mathbf{1}$. Figure 3(b) demonstrates the $R$-module structure of $R_{P_{4}}$ : multiplying $\boldsymbol{p}$ by an arbitrary element $r \in R$ produces the spline $r \boldsymbol{p}=\left(r \boldsymbol{p}_{v_{1}}, r \boldsymbol{p}_{v_{2}}, r \boldsymbol{p}_{v_{3}}\right) \in R_{P_{4}}$.

One major question we study is whether there are nontrivial generalized splines.

Definition 2.5. A nontrivial generalized spline is an element $\boldsymbol{p} \in R_{G}$ that is not in the principal ideal $R \mathbf{1}$.

In other words, we ask whether the $R$-module $R_{G}$ contains at least two linearly independent elements. We answer this question completely (and more strongly) in Theorem 5.1 and Corollary 5.6: yes, except in the trivial cases when $G$ consists of a single point or $R$ is zero.

If some edge labels were zero then the ring of splines could be trivial for trivial algebraic reasons: for instance, if all edge labels of $G$ are zero then the only elements of $R_{G}$ are trivial splines. This is why $\alpha(e)$ is always nonzero in Definition 2.1. 
Definition 2.6. Let $(G, \alpha)$ and $\left(G^{\prime}, \alpha^{\prime}\right)$ be edge-labeled graphs with respect to $R$. A homomorphism of edge-labeled graphs $\phi:(G, \alpha) \rightarrow\left(G^{\prime}, \alpha^{\prime}\right)$ is a graph homomorphism $\phi_{1}: G \rightarrow G^{\prime}$ together with a ring automorphism $\phi_{2}: R \rightarrow R$ so that for each edge $e \in E_{G}$ we have $\phi_{2}(\alpha(e))=\alpha^{\prime}\left(\phi_{1}(e)\right)$ :

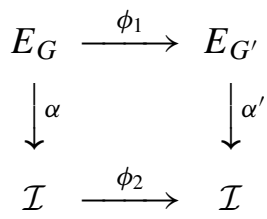

An isomorphism of edge-labeled graphs is a homomorphism of edge-labeled graphs whose underlying graph homomorphism is in fact an isomorphism.

We stress that the map $\phi_{2}$ is a ring automorphism. This ensures that $\phi_{2}$ induces a map on the set of ideals $\phi_{2}: \mathcal{I} \rightarrow \mathcal{I}$ and that the diagram in (1) is well defined. The content of the definition is that the diagram commutes.

Many interesting homomorphisms of edge-labeled graphs arise when $\phi_{2}: R \rightarrow R$ is the identity homomorphism. Indeed, when $R$ is the integers, this is essentially the only case. However, some rings $R$ have very interesting automorphisms: for instance, when $R$ is a polynomial on $n$ variables, the symmetric group on $n$ letters acts on $R$ by permuting variables. This induces an important action in equivariant cohomology, which is substantively different from a closely related action induced by the identity ring automorphism [Tymoczko 2008]. Our first proposition confirms that the ring of generalized splines is invariant under edge-labeled isomorphisms. More precisely, when two graphs are edge-labeled isomorphic, any generalized spline for one graph will be a generalized spline for the other.

Proposition 2.7. If $\phi:(G, \alpha) \rightarrow\left(G^{\prime}, \alpha^{\prime}\right)$ is an isomorphism of edge-labeled graphs then $\phi$ induces an isomorphism of the corresponding rings of generalized splines $\phi_{*}: R_{G} \cong R_{G^{\prime}}$ according to the rule that $\phi_{*}(\boldsymbol{p})_{\phi_{1}(u)}=\phi_{2}\left(\boldsymbol{p}_{u}\right)$ for each $u \in V_{G}$.

Proof. By definition of generalized splines,

$$
\boldsymbol{p} \in R_{G} \Longleftrightarrow \boldsymbol{p}_{u}-\boldsymbol{p}_{v} \in \alpha(e) \text { for each edge } e=u v \text { in } E_{G} .
$$

The map $\phi_{2}: R \rightarrow R$ is an automorphism of rings, so the GKM conditions imply

$$
\boldsymbol{p} \in R_{G} \Longleftrightarrow \phi_{2}\left(\boldsymbol{p}_{u}\right)-\phi_{2}\left(\boldsymbol{p}_{v}\right) \in \phi_{2}(\alpha(e)) \text { for each edge } e=u v \text { in } E_{G} .
$$

The map $\phi_{1}$ is an isomorphism between the underlying graphs $G$ and $G^{\prime}$, so $e$ is an edge in $G$ if and only if $\phi_{1}(e)$ is an edge in $G^{\prime}$. Incorporating the fact that $\alpha^{\prime}\left(\phi_{1}(e)\right)=\phi_{2}(\alpha(e))$ for each edge $e \in E_{G}$, this means (2) is equivalent to

$$
\phi_{2}\left(\boldsymbol{p}_{u}\right)-\phi_{2}\left(\boldsymbol{p}_{v}\right) \in \alpha^{\prime}\left(\phi_{1}(e)\right) \text { for each edge } e^{\prime}=\phi_{1}(u) \phi_{1}(v) \text { in } E_{G^{\prime}} .
$$


We have that (3) is equivalent to $\phi_{*}(\boldsymbol{p}) \in R_{G^{\prime}}$, so we conclude that $\boldsymbol{p}$ is a generalized spline in $R_{G}$ if and only if $\phi_{*}(\boldsymbol{p})$ is in $R_{G^{\prime}}$.

The next proposition verifies that a generalized spline for the pair $(G, \alpha)$ is a generalized spline for every subgraph of $G$.

Proposition 2.8. Let $(G, \alpha)$ be an edge-labeled graph and $G^{\prime}=\left(V^{\prime}, E^{\prime}\right)$ a subgraph of $G$. Let $\left(G^{\prime},\left.\alpha\right|_{E^{\prime}}\right)$ be the edge-labeled graph whose function $\left.\alpha\right|_{E^{\prime}}$ denotes the restriction of $\alpha$ to the edge set of $G^{\prime}$. If $\boldsymbol{p}$ is a generalized spline for $(G, \alpha)$ then $\left.\boldsymbol{p}\right|_{V^{\prime}} \in \bigoplus_{v \in V^{\prime}} R$ is a generalized spline for $\left(G^{\prime},\left.\alpha\right|_{E^{\prime}}\right)$.

Proof. Let $G^{\prime} \subseteq G$ as in the hypothesis, let $p$ be a generalized spline for $(G, \alpha)$, and consider the subcollection $\left.\boldsymbol{p}\right|_{V^{\prime}}$ obtained by restricting $\boldsymbol{p}$ to the vertex set $V^{\prime} \subseteq V$ of $G^{\prime}$. For any edge $u v$ in $G^{\prime}$ the corresponding edge $u v$ is in $E$ since $E^{\prime} \subseteq E$. This implies that $\boldsymbol{p}_{u}-\boldsymbol{p}_{v} \in \alpha(u v)$ by the GKM condition for $(G, \alpha)$. Since the edge-labeling function for $G^{\prime}$ is the restriction $\left.\alpha\right|_{E^{\prime}}$ to the edges in $E^{\prime} \subseteq E$, we conclude that the GKM condition is satisfied at every edge of $G^{\prime}$. It follows that $\left.\boldsymbol{p}\right|_{V^{\prime}}$ is a generalized spline for $\left(G^{\prime},\left.\alpha\right|_{E^{\prime}}\right)$.

Example 2.9. Consider the generalized spline on the bold $P_{4}$ in Figure 2(a) with edges labeled as in Figure 1. Removing a leaf and its incident edge from $P_{4}$ gives the subgraph $P_{3}$ in Figure 3(a). The generalized spline for $P_{4}$ still satisfies the GKM condition at every vertex on the subgraph. Thus $\left.\boldsymbol{p}\right|_{P_{3}}$ is a generalized spline for $P_{3}$.

The next proposition shows that the special case when one of the edges is associated to the unit ideal $\alpha(e)=R$ is equivalent to a kind of restriction as in Proposition 2.8. In this case, the edge $e$ can be erased without affecting the ring of generalized splines.

Proposition 2.10. Suppose that the edge-labeled graph $(G, \alpha)$ has an edge e with $\alpha(e)=R$. Let $G^{\prime}=\left(V_{G}, E-\{e\}\right)$ be the graph $G$ with edge e erased, and let $\alpha^{\prime}: E-\{e\} \rightarrow \mathcal{I}$ be the restriction $\alpha^{\prime}=\left.\alpha\right|_{E-\{e\}}$. Then

$$
R_{G}=R_{G^{\prime}} .
$$

Proof. Proposition 2.8 says that every generalized spline of $G$ is a generalized spline of $G^{\prime}$, since $G^{\prime}$ is a subgraph of $G$ with the same vertex set whose labeling agrees on shared edges. Hence $R_{G} \subseteq R_{G^{\prime}}$. To prove the converse, suppose $\boldsymbol{p}$ is a generalized spline for $\left(G^{\prime}, \alpha^{\prime}\right)$. The GKM condition guarantees that $\boldsymbol{p}_{u}-\boldsymbol{p}_{v} \in \alpha(u v)$ for every edge $u v \in E-\{e\}$. In addition, if $u_{0}, v_{0}$ are the endpoints of the edge $e$, then $\boldsymbol{p}_{u_{0}}-\boldsymbol{p}_{v_{0}} \in R$ is vacuously true. Since $\alpha(e)=R$ we conclude that the GKM condition is satisfied for the edge $e$ as well. So $p \in R_{G}$ and $R_{G^{\prime}}=R_{G}$.

We may build generalized splines from disjoint unions of graphs by taking the direct sum of the respective generalized splines. 
Proposition 2.11. If $G=G_{1} \cup G_{2}$ is the union of two disjoint graphs then the ring of splines is $R_{G}=R_{G_{1}} \oplus R_{G_{2}}$.

Proof. Rearranging the GKM conditions gives

$$
\begin{aligned}
R_{G} & =\left\{\boldsymbol{p} \in \bigoplus_{v \in V} R \text { such that } \boldsymbol{p} \text { satisfies the GKM condition at each edge } e \in E(G)\right\} \\
& =\left\{\boldsymbol{p} \in \bigoplus_{v \in V\left(G_{1}\right)} R \text { such that } \boldsymbol{p}_{v}-\boldsymbol{p}_{u} \in \alpha(u v) \text { for all } u v \in E\left(G_{1}\right)\right\} \\
& \oplus\left\{\boldsymbol{p} \in \bigoplus_{v \in V\left(G_{2}\right)} R \text { such that } \boldsymbol{p}_{v}-\boldsymbol{p}_{u} \in \alpha(u v) \text { for all } u v \in E\left(G_{2}\right)\right\} \\
= & R_{G_{1}} \oplus R_{G_{2}}
\end{aligned}
$$

because the vertex sets of $G_{1}$ and $G_{2}$ are disjoint.

Another approach to constructing generalized splines is to build them one vertex at a time. The next result decomposes the $R$-module of generalized splines into a direct sum of the trivial generalized splines and the generalized splines that are zero at a particular vertex.

Theorem 2.12. Suppose that $G$ is a connected graph with edge-labeling function $\alpha: V \rightarrow \mathcal{I}$. Fix a vertex $v \in V$. Then every generalized spline $\boldsymbol{p} \in R_{G}$ can be written uniquely as $\boldsymbol{p}=r \mathbf{1}+\boldsymbol{p}^{v}$ where $\boldsymbol{p}^{v}$ is a generalized spline satisfying $\boldsymbol{p}_{v}^{v}=0$ and $r \in R$ satisfies $r=\boldsymbol{p}_{v}$. In other words, if $M=\left\langle\boldsymbol{p}: \boldsymbol{p}_{v}=0\right\rangle$ then $R_{G} \cong R \mathbf{1} \oplus M$ as $R$-modules.

Proof. The trivial generalized spline 1 is in $R_{G}$ by Proposition 2.4. Let $r \in R$ be the element $r=\boldsymbol{p}_{v}$. Then define $\boldsymbol{p}^{v}$ to be the generalized spline $\boldsymbol{p}^{v}=\boldsymbol{p}-r \mathbf{1}$. (There is a unique element in the ring $R_{G}$ that satisfies this equation.) By construction,

$$
\boldsymbol{p}_{v}^{v}=\boldsymbol{p}_{v}-r \mathbf{1}_{v}=r-r=0 .
$$

The previous result could lead us to consider $R$-module bases of generalized splines; see the open questions in Section 7. Instead, we combine it with a result of Rose's to relate the generalized splines that vanish at a particular vertex to the syzygies of the module generated by the edge ideals. (Schumaker also implicitly considered syzygies in an earlier work on splines [1979].)

Corollary 2.13. Suppose $G$ is the dual graph of a hereditary polyhedral complex $\Delta$ and that $R$ is the polynomial ring $\mathbb{R}\left[x_{1}, x_{2}, \ldots, x_{d}\right]$. For each edge $e$ in $G$, let $\ell_{e}$ be an affine form generating the polynomials vanishing on the intersection of faces in $\Delta$ corresponding to $e$. Define $\alpha$ to be the function $\alpha(e)=\left\langle\ell_{e}^{r+1}\right\rangle$ for each edge $e$ and let

$$
B=\left\{\left(b_{1}, \ldots, b_{|E|}\right) \in R^{|E|}: \text { for all cycles } C \text { in } G,\right.
$$

$$
\text { the linear combination } \left.\sum_{e \in C} b_{e} \ell_{e}^{r+1}=0\right\} \text {. }
$$


Then $M \cong B$ as $R$-modules.

Proof. Under these conditions, Rose proved that $R_{G} \cong R \oplus B$ as $R$-modules [1995, Theorem 2.2]. From the previous claim, we conclude $M \cong B$ as desired.

We close this section by describing the relationship between the ring of generalized splines associated to an edge-labeling $\alpha$ and the ring of generalized splines associated to the edge-labeling $r \alpha$ obtained by scaling.

Theorem 2.14. Suppose that $(G, \alpha)$ is a connected edge-labeled graph. Fix an element $r \in R$ and define the edge-labeling function $r \alpha: E \rightarrow \mathcal{I}$ by $r \alpha(e)=r I_{e}$ for each edge $e \in E$. Choose a vertex $v_{0} \in V$ and define $M=\left\langle\boldsymbol{p}: \boldsymbol{p}_{v_{0}}=0\right\rangle$. If $R$ is an integral domain then

$$
R_{G, r \alpha}=R \mathbf{1} \oplus r M
$$

Proof. Theorem 2.12 showed that $R_{G, \alpha}=R \mathbf{1} \oplus M$. The multiple $r R_{G, \alpha}$ belongs to $R_{G, r \alpha}$ by definition, so $r M \subseteq R_{G, r \alpha}$. We also know the intersection $r M \cap R \mathbf{1}$ is zero since the only element of $R \mathbf{1}$ whose restriction to $v_{0}$ vanishes is the zero spline. So $R_{G, r \alpha} \supseteq R \mathbf{1} \oplus r M$.

We now prove the opposite containment. Suppose $p^{\prime} \in R_{G, r \alpha}$ and suppose $\boldsymbol{p}=\boldsymbol{p}^{\prime}-\boldsymbol{p}_{v_{0}}^{\prime} \mathbf{1}$. (Note that $\boldsymbol{p}$ satisfies the GKM condition for $(G, r \alpha)$ at each edge.) We will prove that $\boldsymbol{p} \in r M$. We split the argument into two pieces: showing that $\boldsymbol{p}$ is divisible by $r$ at each vertex, and then showing that $\boldsymbol{p}$ satisfies the GKM conditions of $r M$.

To begin, we prove by induction that if $v_{k}$ is connected to $v_{0}$ by a path of length $k$ then $\boldsymbol{p}_{v_{k}} \in r R$ is in the principal ideal generated by $r$. The unique path of length zero is our base case, and the element $\boldsymbol{p}_{v_{0}}=0 \in r R$ by construction. Suppose the claim is true for paths of length $k-1$ and let $v_{k}$ be a vertex connected to $v_{0}$ by a path of length $k$. Then $v_{k}$ is adjacent to a vertex $v_{k-1}$ which is connected to $v_{0}$ by a path of length $k-1$. We know $\boldsymbol{p}_{v_{k-1}} \in r R$ by the inductive hypothesis, and $\boldsymbol{p}_{v_{k}}-\boldsymbol{p}_{v_{k-1}} \in r I_{e_{k}}$ for the edge $e_{k}=v_{k-1} v_{k}$ by the GKM condition. The sum $r I_{e_{k}}+r R \subseteq r R$ since ideals are closed under addition, so $\boldsymbol{p}_{v_{k}} \in r R$ as desired. By induction and because $G$ is connected, we conclude that $\boldsymbol{p}_{v} \in r R$ for all $v \in V$.

We just showed that each ring element $\boldsymbol{p}$ is divisible by $r$. For each vertex $v$, let $\boldsymbol{q}_{v}$ be the ring element with $\boldsymbol{p}_{v}=r \boldsymbol{q}_{v}$ and collect the $\boldsymbol{q}_{v}$ into the element $\boldsymbol{q} \in R^{|V|}$. We ask whether $\boldsymbol{q} \in M$. To answer this, we need to know whether for each edge $e=u v$ we have $\boldsymbol{q}_{u}-\boldsymbol{q}_{v} \in I_{e}$. We know that $\boldsymbol{p}_{u}-\boldsymbol{p}_{v} \in r I_{e}$ by the GKM condition. Let $x=\boldsymbol{q}_{u}-\boldsymbol{q}_{v} \in R$ to isolate the underlying algebraic question: If $r x \in r I_{e}$ then is $x \in I_{e}$ ? The answer is yes when $R$ is an integral domain: if $r x \in r I_{e}$ then we can find $y \in I_{e}$ with $r x=r y$. Hence $r(x-y)=0$, which implies $x=y$ as long as $R$ is an integral domain. 


\section{The GKM matrix}

The results in the previous section allow us to build new generalized splines from existing ones. To construct generalized splines from scratch we need a systematic method for recording and analyzing GKM conditions. We do this by representing GKM conditions in matrix form. This section shows how to construct GKM matrices and gives several examples.

Our definition of the GKM matrix assumes the graph $G$ is directed. Remark 3.5 shows that changing the directions on the edges of $G$ does not affect the solution space of the matrix, so we generally omit orientations from our figures and our discussion.

Definition 3.1. The GKM matrix of the directed, edge-labeled graph $(G, \alpha)$ is an $|E| \times|V|$ matrix constructed so that the row corresponding to each directed edge $e=u v \in E$ has

- 1 in the column corresponding to $u$,

- -1 in the column corresponding to $v$, and

- 0 otherwise.

An extended GKM matrix of the pair $(G, \alpha)$ is an $|E| \times(|V|+1)$ matrix whose first $|V|$ columns are the GKM matrix, and whose last entry in the row corresponding to edge $e$ is any element $\alpha_{e} \in \alpha(e)$. When there is no risk of confusion, we refer to an extended GKM matrix as simply the GKM matrix.

For instance, if $\alpha(e)=\left\langle\alpha_{e_{1}}, \ldots, \alpha_{e_{m}}\right\rangle$ is finitely generated, we could write the last entry in the row corresponding to $e$ as $q_{e_{1}} \alpha_{e_{1}}+\cdots+q_{e_{m}} \alpha_{e_{m}}$ for arbitrary $q_{e_{i}} \in R$. In particular, if the ideal $\alpha(e)$ is principal and $\alpha(e)=\left\langle\alpha_{e}\right\rangle$ then we typically write the last column of the extended GKM matrix as the vector $\left(q_{e} \alpha_{e}\right)_{e \in E}$ for arbitrary coefficients $q_{e} \in R$.

Remark 3.2. Using this language, we can reframe the syzygy module of spline ideals that Rose defined and that we saw in Corollary 2.13. (See also [Schumaker 1979].) In our context, the syzygy module is essentially the collection of elements $q_{e} \in \alpha(e)$ from the edge ideals so that $\sum_{e \in C} q_{e}=0$ for each cycle $C$ in $G$. In other words, it describes a collection of elements $q_{e} \in \alpha(e)$ for which the extended GKM matrix represents a homogeneous system of equations. This condition appears naturally as we analyze the ring $R_{G}$ further in Theorem 6.3.

Generally we consider $q_{e}$ to be a parameter that takes values in $R$, as in the following proposition, which follows immediately from the construction of the GKM matrix. 
Proposition 3.3. Let $M_{G}$ denote the GKM matrix of $(G, \alpha)$. Then the spline $\boldsymbol{p} \in R^{|V|}$ is a generalized spline for $(G, \alpha)$ if and only if there is an extended GKM matrix $\left[M_{G} \mid \boldsymbol{v}\right]$ for which $\boldsymbol{p}$ is a solution.

Proof. The matrix $M_{G}$ is constructed to record the GKM condition at every edge $e_{i, j} \in E(G)$. Hence a spline $\boldsymbol{p}=\left(\boldsymbol{p}_{v_{1}}, \ldots, \boldsymbol{p}_{v_{|V|}}\right) \in R^{|V|}$ is a generalized spline for $(G, \alpha)$ if and only if $M_{G} \boldsymbol{p}=\boldsymbol{v}$ for some vector $\boldsymbol{v}=\left(\alpha_{e}\right)_{e \in E}$. This is equivalent to saying the spline $\boldsymbol{p}$ is a solution to the system $\left[M_{G} \mid v\right]$ for some extended GKM matrix, as claimed.

We can now manipulate $M_{G}$ to obtain systems of equations that are equivalent to the original GKM conditions on $G$. We state the following corollary simply to stress this fundamental linear algebra property.

Corollary 3.4. If $\left[M^{\prime} \mid \boldsymbol{v}^{\prime}\right]$ is obtained from $[M \mid \boldsymbol{v}]$ by a series of reversible row or column operations, then the solution set in $R^{|V|}$ to $\left[M^{\prime} \mid \boldsymbol{v}^{\prime}\right]$ is the same as that of $[M \mid \boldsymbol{v}]$.

Reversible operations correspond to invertible matrices in $\mathrm{GL}_{|V|}(R)$. For instance, multiplying a row by $x$ is not reversible for the ring $R=\mathbb{C}[x]$ since $1 / x$ is not in $R$. However, multiplying a row by $x$ is reversible when $R=\mathbb{C}(x)$.

Remark 3.5. Changing the direction of a given edge in $G$ amounts to multiplying the corresponding row in $M_{G}$ by -1 , a reversible operation. Hence while the definition of the GKM matrix for the pair $(G, \alpha)$ requires a directed graph, the actual direction chosen is irrelevant to the solution set given by Proposition 3.3.

Example 3.6. We start with the path $P_{3}$ from Figure 3(a). Its extended GKM matrix is

$$
M_{P_{3}}=\left[\begin{array}{rrr|r}
1 & -1 & 0 & q_{1} \alpha_{1} \\
0 & 1 & -1 & q_{2} \alpha_{2}
\end{array}\right],
$$

whose rows may be added to obtain the equivalent system

$$
\left[\begin{array}{lll|l}
1 & 0 & -1 & q_{2} \alpha_{2}+q_{1} \alpha_{1} \\
0 & 1 & -1 & q_{2} \alpha_{2}
\end{array}\right]
$$

If $\boldsymbol{p}=\left(\boldsymbol{p}_{v_{1}}, \boldsymbol{p}_{v_{2}}, \boldsymbol{p}_{v_{3}}\right) \in R_{P_{3}}$ then the system has dependent variables $\boldsymbol{p}_{v_{1}}$ and $\boldsymbol{p}_{v_{2}}$ and independent variable $\boldsymbol{p}_{v_{3}}$. All solutions may be written in the form

$$
\begin{aligned}
& \boldsymbol{p}_{v_{1}}=\boldsymbol{p}_{v_{3}}+q_{2} \alpha_{2}+q_{1} \alpha_{1}, \\
& \boldsymbol{p}_{v_{2}}=\boldsymbol{p}_{v_{3}}+q_{2} \alpha_{2},
\end{aligned}
$$

where $\boldsymbol{p}_{v_{3}}, q_{1}$, and $q_{2}$ are freely chosen elements of $R$. Setting $\boldsymbol{p}_{v_{3}}=0, q_{1}=1$, and $q_{2}=1$ yields the generalized spline in Figure 3(a).

The following generalization will be a central part of our proof of Theorem 3.8. 
Example 3.7. Consider the path $P_{n}$ on $n$ vertices:

$$
P_{n}=\underset{v_{1}}{\bullet} \underset{v_{2}}{\bullet} \cdots \underset{v_{n-1}}{\bullet} \stackrel{\alpha\left(e_{1,2}\right)}{\bullet} \underset{v_{n}}{\bullet}
$$

The GKM matrix for this path is

$$
\left[\begin{array}{rrrrrrr|c}
1 & -1 & 0 & 0 & \cdots & 0 & 0 & \alpha_{1,2} \\
0 & 1 & -1 & 0 & \cdots & 0 & 0 & \alpha_{2,3} \\
0 & 0 & 1 & -1 & \cdots & 0 & 0 & \alpha_{3,4} \\
\vdots & \vdots & \vdots & \vdots & \ddots & \vdots & \vdots & \vdots \\
0 & 0 & 0 & 0 & \cdots & 1 & -1 & \alpha_{n-1, n}
\end{array}\right]
$$

where $\alpha_{i, i+1} \in \alpha\left(e_{i, i+1}\right)$ are arbitrarily chosen. As before, we can row-reduce the GKM matrix by setting row $i$ to be the sum $\sum_{k=i}^{n}$ (row $k$ ) for each $1 \leq i \leq n$. We obtain an equivalent system of rank $n-1$ in which $\boldsymbol{p}_{v_{n}}$ is the only free variable in the set $\left\{\boldsymbol{p}_{v_{i}}: i=1, \ldots, n\right\}$. (This system is of maximal rank since an $(n-1) \times(n+1)$ system of equations can have at most one free variable among the $\boldsymbol{p}_{v_{i}}$.) Figure 4 shows this equivalent system:

$$
\left[\begin{array}{ccccccr|c}
1 & 0 & 0 & 0 & \cdots & 0 & -1 & \alpha_{n-1, n}+\cdots+\alpha_{3,4}+\alpha_{2,3}+\alpha_{1,2} \\
0 & 1 & 0 & 0 & \cdots & 0 & -1 & \alpha_{n-1, n}+\cdots+\alpha_{3,4}+\alpha_{2,3} \\
0 & 0 & 1 & 0 & \cdots & 0 & -1 & \alpha_{n-1, n}+\cdots+\alpha_{3,4} \\
\vdots & \vdots & \vdots & \vdots & \ddots & \vdots & \vdots & \vdots \\
0 & 0 & 0 & 0 & \cdots & 1 & -1 & \alpha_{n-1, n}
\end{array}\right]
$$

Figure 4. A system equivalent to the GKM matrix for $P_{n}$.

The linear combinations that occur in the last column of the matrix in Figure 4 can be used to construct generalized splines for more complicated graphs as well. For instance, the next result builds on this description of paths to describe a collection of (usually) nontrivial generalized splines for the cycle $C_{n}$.

Theorem 3.8. Let $C_{n}$ be a finite edge-labeled cycle given by vertices $v_{1}, v_{2}, \ldots, v_{n}$ in order. Define the vector $\boldsymbol{p} \in R^{|V|}$ by

$$
\left[\begin{array}{c}
\boldsymbol{p}_{v_{1}} \\
\boldsymbol{p}_{v_{2}} \\
\boldsymbol{p}_{v_{3}} \\
\vdots \\
\boldsymbol{p}_{v_{n-1}} \\
\boldsymbol{p}_{v_{n}}
\end{array}\right]=\boldsymbol{p}_{v_{1}}\left[\begin{array}{c}
1 \\
1 \\
1 \\
\vdots \\
1 \\
1
\end{array}\right]+\alpha_{1, n}\left[\begin{array}{ccccc}
0 & 0 & \cdots & 0 & 0 \\
1 & 0 & \cdots & 0 & 0 \\
1 & 1 & \cdots & 0 & 0 \\
\vdots & \vdots & \ddots & \vdots & \vdots \\
1 & 1 & \cdots & 1 & 0 \\
1 & 1 & \cdots & 1 & 1
\end{array}\right]\left[\begin{array}{c}
\alpha_{1,2} \\
\alpha_{2,3} \\
\alpha_{3,4} \\
\vdots \\
\alpha_{n-2, n-1} \\
\alpha_{n-1, n}
\end{array}\right]
$$


with arbitrary choices of $\boldsymbol{p}_{v_{1}} \in R, \alpha_{i, i+1} \in \alpha\left(e_{i, i+1}\right)$, and $\alpha_{1, n} \in \alpha\left(e_{1, n}\right)$. Then $\boldsymbol{p}$ is a generalized spline for $C_{n}$. The spline $\boldsymbol{p}$ is nontrivial exactly when $\alpha_{1, n}$ and at least one of the $\alpha_{i, i+1}$ are nonzero.

Proof. We check that $\boldsymbol{p} \in R^{n}$ satisfies the GKM condition at every edge of $C_{n}$. For all $i$ with $2 \leq i \leq n-1$ we have

$$
\begin{aligned}
& \boldsymbol{p}_{v_{i+1}}-\boldsymbol{p}_{v_{i}} \\
& \quad=\left(\boldsymbol{p}_{v_{1}}+\alpha_{1, n}\left(\alpha_{1,2}+\cdots+\alpha_{i-1, i}+\alpha_{i, i+1}\right)\right)-\left(\boldsymbol{p}_{v_{1}}+\alpha_{1, n}\left(\alpha_{1,2}+\cdots+\alpha_{i-1, i}\right)\right) \\
& \quad=\alpha_{1, n} \alpha_{i, i+1},
\end{aligned}
$$

which is in $\alpha\left(e_{i, i+1}\right)$ by assumption on $\alpha_{i, i+1}$. It remains to check that the GKM condition is satisfied at edges $e_{1,2}$ and $e_{1, n}$. At edge $e_{1,2}$ we have

$$
\boldsymbol{p}_{v_{2}}-\boldsymbol{p}_{v_{1}}=\left(\boldsymbol{p}_{v_{1}}+\alpha_{1, n} \alpha_{1,2}\right)-\boldsymbol{p}_{v_{1}}=\alpha_{1, n} \alpha_{1,2},
$$

which is in the ideal $\alpha\left(e_{1,2}\right)$. At edge $e_{1, n}$ we have

$$
\boldsymbol{p}_{v_{n}}-\boldsymbol{p}_{v_{1}}=\left(\boldsymbol{p}_{v_{1}}+\alpha_{1, n}\left(\alpha_{1,2}+\cdots+\alpha_{n-1, n}\right)\right)-\boldsymbol{p}_{v_{1}}=\alpha_{1, n}\left(\alpha_{1,2}+\cdots+\alpha_{n-1, n}\right),
$$

which is in the ideal $\alpha\left(e_{1, n}\right)$. Hence $\boldsymbol{p}$ is a generalized spline for $C_{n}$. The spline $\boldsymbol{p}$ is nontrivial if and only if the second term is nonzero, namely, when $\alpha_{1, n}$ and at least one of the $\alpha_{i, i+1}$ are nonzero.

Theorem 3.8 actually does more: it identifies a collection of generalized splines for $C_{n}$ that are linearly independent for many choices of $R$. Indeed, we can write the generalized splines from Theorem 3.8 in parametric form:

$$
\left[\begin{array}{c}
\boldsymbol{p}_{v_{1}} \\
\boldsymbol{p}_{v_{2}} \\
\boldsymbol{p}_{v_{3}} \\
\boldsymbol{p}_{v_{4}} \\
\vdots \\
\boldsymbol{p}_{v_{n}}
\end{array}\right]=\boldsymbol{p}_{v_{1}}\left[\begin{array}{c}
1 \\
1 \\
1 \\
1 \\
\vdots \\
1
\end{array}\right]+\alpha_{1, n} \alpha_{1,2}\left[\begin{array}{c}
0 \\
1 \\
1 \\
1 \\
\vdots \\
1
\end{array}\right]+\alpha_{1, n} \alpha_{2,3}\left[\begin{array}{c}
0 \\
0 \\
1 \\
1 \\
\vdots \\
1
\end{array}\right]+\cdots+\alpha_{1, n} \alpha_{n-1, n}\left[\begin{array}{c}
0 \\
0 \\
0 \\
\vdots \\
1
\end{array}\right]
$$

with coefficients $\boldsymbol{p}_{v_{1}} \in R$ and $\alpha_{i, i+1} \in \alpha\left(e_{i, i+1}\right)=I_{i, i+1}$ for all $1 \leq i \leq n-1$. The vectors $[1,1,1, \ldots, 1]^{T},[0,1,1, \ldots, 1]^{T}, \ldots,[0,0,0, \ldots, 1]^{T}$ are linearly independent in $R^{n}$ but are not necessarily elements of $R_{C_{n}}$. If $R$ is an integral domain then for any fixed choices of $\alpha_{i, j} \in \alpha\left(e_{i, j}\right)=I_{i, j}$ the vectors $[1,1,1, \ldots, 1]^{T}$, $\alpha_{1, n} \alpha_{1,2}[0,1,1, \ldots, 1]^{T}, \ldots, \alpha_{1, n} \alpha_{n-1, n}[0,0,0, \ldots, 1]^{T}$ are both linearly independent and in $R_{C_{n}}$.

We will use these kinds of splines - which arise naturally when considering the GKM matrix — repeatedly in subsequent sections of the paper. 


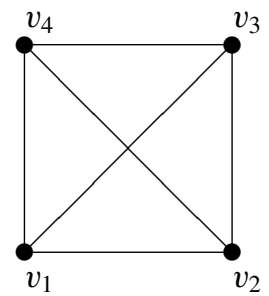

$$
\begin{aligned}
& \boldsymbol{p}_{v_{1}}-\boldsymbol{p}_{v_{2}} \in \alpha\left(e_{1,2}\right)=\left\langle\alpha_{1,2}\right\rangle \\
& \boldsymbol{p}_{v_{1}}-\boldsymbol{p}_{v_{3}} \in \alpha\left(e_{1,3}\right)=\left\langle\alpha_{1,3}\right\rangle \\
& \boldsymbol{p}_{v_{1}}-\boldsymbol{p}_{v_{4}} \in \alpha\left(e_{1,4}\right)=\left\langle\alpha_{1,4}\right\rangle \\
& \boldsymbol{p}_{v_{2}}-\boldsymbol{p}_{v_{3}} \in \alpha\left(e_{2,3}\right)=\left\langle\alpha_{2,3}\right\rangle \\
& \boldsymbol{p}_{v_{2}}-\boldsymbol{p}_{v_{4}} \in \alpha\left(e_{2,4}\right)=\left\langle\alpha_{2,4}\right\rangle \\
& \boldsymbol{p}_{v_{3}}-\boldsymbol{p}_{v_{4}} \in \alpha\left(e_{3,4}\right)=\left\langle\alpha_{3,4}\right\rangle
\end{aligned}
$$

Figure 5. GKM conditions for $K_{4}$ whose ideals are all principal.

$$
M_{K_{4}}=\left[\begin{array}{rrrr|l}
1 & 0 & 0 & -1 & q_{1,4} \alpha_{1,4} \\
0 & 1 & 0 & -1 & q_{2,3} \alpha_{2,3} \\
0 & 0 & 1 & -1 & q_{3,4} \alpha_{3,4} \\
0 & 0 & 0 & 0 & q_{1,2} \alpha_{1,2}-q_{1,4} \alpha_{1,4}+q_{2,4} \alpha_{2,4} \\
0 & 0 & 0 & 0 & q_{1,3} \alpha_{1,3}-q_{1,4} \alpha_{1,4}+q_{3,4} \alpha_{3,4} \\
0 & 0 & 0 & 0 & q_{2,3} \alpha_{2,3}-q_{2,4} \alpha_{2,4}+q_{3,4} \alpha_{3,4}
\end{array}\right]
$$

Figure 6. A system equivalent to the extended GKM matrix for $K_{4}$ when all ideals are principal.

Example 3.9. We return to the case of the complete graph $K_{4}$ whose ideals $\alpha(e)$ are all principal. By Definition 2.3, the tuple $\boldsymbol{p}=\left(\boldsymbol{p}_{v_{1}}, \boldsymbol{p}_{v_{2}}, \boldsymbol{p}_{v_{3}}, \boldsymbol{p}_{v_{4}}\right)$ is a generalized spline for $K_{4}$ if and only if it satisfies the GKM conditions in Figure 5.

The difference $\boldsymbol{p}_{v_{i}}-\boldsymbol{p}_{v_{j}}$ is in the ideal $\alpha\left(e_{i, j}\right)=\left\langle\alpha_{i, j}\right\rangle$ if and only if the difference $\boldsymbol{p}_{v_{i}}-\boldsymbol{p}_{v_{j}}=q_{i, j} \alpha_{i, j}$ for some $q_{i, j} \in R$, so we represent these GKM conditions by the following matrix equation (the coefficient matrix is the GKM matrix):

$$
\left[\begin{array}{rrrr}
1 & -1 & 0 & 0 \\
1 & 0 & -1 & 0 \\
1 & 0 & 0 & -1 \\
0 & 1 & -1 & 0 \\
0 & 1 & 0 & -1 \\
0 & 0 & 1 & -1
\end{array}\right]\left[\begin{array}{l}
\boldsymbol{p}_{v_{1}} \\
\boldsymbol{p}_{v_{2}} \\
\boldsymbol{p}_{v_{3}} \\
\boldsymbol{p}_{v_{4}}
\end{array}\right]=\left[q_{1,2}, q_{1,3}, q_{1,4}, q_{2,3}, q_{2,4}, q_{3,4}\right]\left[\begin{array}{c}
\alpha_{1,2} \\
\alpha_{1,3} \\
\alpha_{1,4} \\
\alpha_{2,3} \\
\alpha_{2,4} \\
\alpha_{3,4}
\end{array}\right] .
$$

After several invertible row operations in which we add various rows to other rows, we obtain an equivalent system of equations such as that given in Figure 6.

\section{Generalized splines for trees}

We will now use the GKM matrix to describe all generalized splines for trees. We start by describing the generalized splines for paths, using the same argument as that for trees but without the notational technicalities. 
Figure 4 shows a matrix that is row-equivalent to the GKM matrix for the path $\left(P_{n}, \alpha\right)$. The solutions can be written in parametric form as

$$
\left[\begin{array}{c}
\boldsymbol{p}_{v_{1}} \\
\boldsymbol{p}_{v_{2}} \\
\boldsymbol{p}_{v_{3}} \\
\boldsymbol{p}_{v_{4}} \\
\vdots \\
\boldsymbol{p}_{v_{n-1}} \\
\boldsymbol{p}_{v_{n}}
\end{array}\right]=\boldsymbol{p}_{v_{n}}\left[\begin{array}{c}
1 \\
1 \\
1 \\
1 \\
\vdots \\
1 \\
1
\end{array}\right]+\alpha_{n-1, n}\left[\begin{array}{c}
1 \\
1 \\
1 \\
1 \\
\vdots \\
1 \\
0
\end{array}\right]+\cdots+\alpha_{3,4}\left[\begin{array}{c}
1 \\
1 \\
1 \\
0 \\
\vdots \\
0 \\
0
\end{array}\right]+\alpha_{2,3}\left[\begin{array}{c}
1 \\
1 \\
0 \\
0 \\
\vdots \\
0 \\
0
\end{array}\right]+\alpha_{1,2}\left[\begin{array}{c}
1 \\
0 \\
0 \\
0 \\
\vdots \\
0 \\
0
\end{array}\right],
$$

where the coefficients $\boldsymbol{p}_{v_{n}}$ and $\alpha_{i, i+1}$ for all $1 \leq i \leq n-1$ are chosen arbitrarily from the sets $R$ and $\alpha\left(e_{i, i+1}\right)=I_{i, i+1}$ respectively. By Corollary 3.4, this gives precisely the collection of generalized splines for the path $P_{n}$.

When $R$ is an integral domain, this also gives linearly independent vectors in $R_{P_{n}}$ (for any choices of $\alpha_{i, i+1} \in I_{i, i+1}$ ):

$$
\mathcal{B}_{R_{P_{n}}}=\left\{\left[\begin{array}{c}
1 \\
1 \\
1 \\
1 \\
\vdots \\
1 \\
1
\end{array}\right],\left[\begin{array}{c}
\alpha_{n-1, n} \\
\alpha_{n-1, n} \\
\alpha_{n-1, n} \\
\alpha_{n-1, n} \\
\vdots \\
\alpha_{n-1, n} \\
0
\end{array}\right], \ldots,\left[\begin{array}{c}
\alpha_{3,4} \\
\alpha_{3,4} \\
\alpha_{3,4} \\
0 \\
\vdots \\
0 \\
0
\end{array}\right],\left[\begin{array}{c}
\alpha_{2,3} \\
\alpha_{2,3} \\
0 \\
0 \\
\vdots \\
0 \\
0
\end{array}\right],\left[\begin{array}{c}
\alpha_{1,2} \\
0 \\
0 \\
0 \\
\vdots \\
0 \\
0
\end{array}\right]\right\}
$$

Morally speaking, this decomposition describes something very close to a basis for the generalized splines - as long as we can write a basis for the ideals $I_{i, i+1}$. For instance, when each ideal $I_{i, i+1}$ is principal and $\alpha_{i, i+1}$ denotes the generator of $I_{i, i+1}$ for each $1 \leq i \leq n-1$, then these vectors form a basis for $R_{P_{n}}$. In general, we won't be able to find a basis for $R_{G}$ because we can't even necessarily find bases for the ideals $I_{i, i+1}$. Even when $R$ is a polynomial ring, we need all of the technical tools developed in the theory of Gröbner bases to compute bases of ideals in $R$.

However, we can find generators for the splines on trees. We reformulate the essential property of this basis from the point of view of trees. Observe that $\boldsymbol{p} \in R_{P_{n}}$ must satisfy the following property for any $v_{i}, v_{j} \in V\left(P_{n}\right)$ with $i<j$ :

$$
\boldsymbol{p}_{v_{j}}=\boldsymbol{p}_{v_{i}}+\sum_{k=i}^{j-1} \alpha_{k, k+1} \quad \text { for some } \alpha_{k, k+1} \in I_{k, k+1} \text {. }
$$

Trees are more complicated than paths, so describing the general result precisely is more complicated. The main idea is similar to the one above, though. It relies on the fact that there is exactly one path between any two vertices in a tree, as well as on (7). 
Theorem 4.1. Let $T=(V, E, \alpha)$ be a finite edge-labeled tree. The tuple $\boldsymbol{p} \in R^{|T|}$ is a generalized spline $\boldsymbol{p} \in R_{T}$ if and only if given any two vertices $v_{i}, v_{j} \in V$ we may write

$$
\boldsymbol{p}_{v_{j}}=\boldsymbol{p}_{v_{i}}+\alpha_{i, i_{1}}+\cdots+\alpha_{i_{m-1}, i_{m}}+\alpha_{i_{m}, j} \quad \text { for some } \alpha_{l, k} \in \alpha\left(e_{l, k}\right)=I_{l, k},
$$

where $v_{i}, v_{i_{1}}, \ldots, v_{i_{m}}, v_{j}$ are the vertices in the unique path connecting $v_{i}$ and $v_{j}$ in the tree T. Furthermore $\boldsymbol{p}$ is nontrivial if and only if at least one of the $\alpha_{l, k}$ is nonzero.

Proof. We proceed via induction on $|V|$. The base case $|V|=1$ is trivial since $E=\varnothing$. We also prove the case $|V|=2$, namely, when $T$ is a path on two vertices. Denote the vertices of $T$ by $v_{1}$ and $v_{2}$ and the edge set by $E=\left\{e_{1,2}\right\}$. Now let $\boldsymbol{p}=\left(p_{v_{1}}, p_{v_{2}}\right) \in R^{2}$. By Definition 2.3 we know $\boldsymbol{p} \in R_{T}$ if and only if $\boldsymbol{p}_{v_{1}}-\boldsymbol{p}_{v_{2}} \in I_{1,2}$. We rewrite this as $\boldsymbol{p}_{v_{1}}=\boldsymbol{p}_{v_{2}}+\alpha_{1,2}$ for some choice of $\alpha_{1,2} \in I_{1,2}$. In other words $\boldsymbol{p}$ is a generalized spline for $T$ if and only if $\boldsymbol{p}$ satisfies (8) for all pairs of vertices in $V=\left\{v_{1}, v_{2}\right\}$. Furthermore $\boldsymbol{p}$ is nontrivial if and only if $\boldsymbol{p}_{v_{1}} \neq \boldsymbol{p}_{v_{2}}$ or equivalently $\alpha_{1,2} \neq 0$.

Assume the theorem holds for every tree with at most $n$ vertices and let $T^{\prime}=$ $\left(V^{\prime}, E^{\prime}, \alpha\right)$ with $\left|V^{\prime}\right|=n+1$. Suppose $\boldsymbol{p} \in R^{\left|V^{\prime}\right|}$ satisfies (8) for all pairs of vertices in $V^{\prime}$ and let $e_{h, k} \in E^{\prime}$ be an arbitrary edge. Since $v_{h}$ and $v_{k}$ are adjacent in $T^{\prime}$ we know $\boldsymbol{p}_{k}=\boldsymbol{p}_{h}+\alpha_{h, k}$ for some $\alpha_{h, k} \in I_{h, k}$ by (8). Rewriting this condition, we obtain $\boldsymbol{p}_{k}-\boldsymbol{p}_{h} \in I_{h, k}$. Since $e_{h, k}$ was arbitrary we conclude $\boldsymbol{p} \in R_{T^{\prime}}$.

Conversely, suppose that $\boldsymbol{p} \in R_{T^{\prime}}$. We show that $\boldsymbol{p}$ satisfies (8) for all vertices in $V^{\prime}$. Without loss of generality, label the vertices of $T^{\prime}$ so that $v_{n+1}$ is a leaf adjacent to $v_{n}$. Choose arbitrary $v_{i}, v_{j} \in V^{\prime}$ and let $v_{i}, v_{i_{1}}, \ldots, v_{i_{m}}, v_{j}$ denote the vertices in the unique path connecting $v_{i}$ and $v_{j}$ in $T^{\prime}$. Let $T$ denote the subgraph $T \subseteq T^{\prime}$ induced by $v_{i}, v_{i_{1}}, \ldots, v_{i_{m}}, v_{j}$. The graph $T$ is a tree itself, since it is a connected subgraph of a tree. The restriction of $\boldsymbol{p}$ to the vertices in $T$ is a generalized spline for $T$ by Proposition 2.8. If $T$ has at most $n$ vertices then the inductive hypothesis implies that $\boldsymbol{p}$ satisfies (8) for the pair $v_{i}, v_{j}$. If $T$ has $n+1$ vertices then $T$ is a path of length $n+1$. Figure 4 shows a system equivalent to the GKM matrix in this case. The first row of this matrix describes the equation

$$
\boldsymbol{p}_{v_{j}}=\boldsymbol{p}_{v_{i}}+\alpha_{i, i_{1}}+\cdots+\alpha_{i_{m-1}, i_{m}}+\alpha_{i_{m}, j}
$$

for some set $\alpha_{l, k} \in \alpha\left(e_{l, k}\right)=I_{l, k}$. In other words, this graph also satisfies (8), proving our claim.

Finally, the spline $\boldsymbol{p}$ is nontrivial if and only if there exists some pair of vertices $v_{i}, v_{j} \in V^{\prime}$ such that $\boldsymbol{p}_{v_{i}} \neq \boldsymbol{p}_{v_{j}}$. This is equivalent to saying that the coefficients $\alpha_{i, i_{1}}, \alpha_{i_{1}, i_{2}}, \ldots, \alpha_{i_{m-1}, i_{m}}, \alpha_{i_{m}, j}$ associated to the path $v_{i}, v_{i_{1}}, \ldots, v_{i_{m}}, v_{j}$ are not all equal to 0 , by (8). Equivalently there exists a pair $l, k$ with $\alpha_{l, k} \neq 0$ as desired. 


\section{Existence of generalized splines and lower bounds on the rank of $\boldsymbol{R}_{\boldsymbol{G}}$}

We now address a fundamental question: Do nontrivial generalized splines exist for an arbitrary edge-labeled graph $(G, \alpha)$ ? We solved this question in the case of edge-labeled cycles $\left(C_{n}, \alpha\right)$ in Theorem 3.8. The answer in that case (yes) leads naturally to a stronger result: Equation (5) actually identifies a collection of generalized splines that are linearly independent when $R$ is an integral domain. The condition that $R$ be an integral domain is crucial, as Bowden and Tymoczko show in forthcoming work [2015].

Similarly, we will answer the existence question for generalized splines on arbitrary $(G, \alpha)$ (yes, unless $G$ consists of a single vertex) by constructing a collection of generalized splines that are linearly independent when $R$ is an integral domain. This provides a lower bound on the rank of $R_{G}$ as an $R$-module when $R_{G}$ is a free $R$-module, and constructs a collection of generators associated to vertices when the ideal $\alpha(e)$ is principal for each edge $e$. All of these hypotheses are satisfied for the generalized splines used to construct equivariant cohomology and equivariant $K$-theory, where constructing bases is an important and well-studied question [Guillemin and Zara 2001; Goldin and Tolman 2009]. Geometrically, Theorem 5.1 and Corollary 5.2 partially extend existing results on flow-up classes in equivariant cohomology, since we broaden the class of varieties for which we can construct linearly independent rank- $n$ collections of flow-up classes. The result is new for equivariant $K$-theory. We note, however, that our flow-up classes are generally not a basis for $R_{G}$.

Corollary 5.2 proves that each $R_{G}$ contains a free submodule of rank $n$ as a special (and simpler) case of Theorem 5.1.

Theorem 5.1. Let $(G, \alpha)$ be a finite edge-labeled graph. Fix any subgraph $G^{\prime}$ of $G$ and let $\boldsymbol{p}$ be a generalized spline for $\left(G^{\prime},\left.\alpha\right|_{G^{\prime}}\right)$. Let $N_{G^{\prime}}=\prod_{S} \alpha_{i, j}$, where each $\alpha_{i, j}$ is a nonzero element of the ideal $\alpha\left(v_{i} v_{j}\right)$ and the product is taken over the set $S$ of edges incident to a vertex in $G^{\prime}$ but not in $G^{\prime}$, namely,

$$
S=\left\{\alpha_{i, j}: v_{i} v_{j} \in E\left(G-G^{\prime}\right) \text { and } v_{i} \in V\left(G^{\prime}\right) \text { or } v_{j} \in V\left(G^{\prime}\right)\right\} .
$$

Then the vector $\boldsymbol{q}$ defined by

$$
\boldsymbol{q}_{v_{i}}= \begin{cases}N_{G^{\prime}} \boldsymbol{p}_{v_{i}} & \text { if } v_{i} \in V\left(G^{\prime}\right), \\ 0 & \text { if } v_{i} \notin V\left(G^{\prime}\right)\end{cases}
$$

is a generalized spline for $G$.

Proof. For each edge $v_{i} v_{j} \in E(G)$, there are three possibilities:

(1) Both $v_{i}$ and $v_{j}$ are in $V\left(G^{\prime}\right)$. Then $\boldsymbol{p}_{v_{i}}-\boldsymbol{p}_{v_{j}}$ satisfies the GKM condition in $G^{\prime}$. Thus $\boldsymbol{q}_{v_{i}}-\boldsymbol{q}_{v_{j}}=N_{G^{\prime}}\left(\boldsymbol{p}_{v_{i}}-\boldsymbol{p}_{v_{j}}\right)$ satisfies the GKM condition for $v_{i}, v_{j}$ in $G$ since $\alpha\left(v_{i} v_{j}\right)$ is an ideal and $N_{G^{\prime}} \in R$. 
(2) Neither $v_{i}$ nor $v_{j}$ is in $V\left(G^{\prime}\right)$. Then the difference $\boldsymbol{q}_{v_{i}}-\boldsymbol{q}_{v_{j}}=0-0$ vacuously satisfies the GKM condition for $v_{i}, v_{j}$ in $G$.

(3) Exactly one of $v_{i}, v_{j}$ is in $V\left(G^{\prime}\right)$. Suppose that $v_{i} \in V\left(G^{\prime}\right)$ and $v_{j} \notin V\left(G^{\prime}\right)$. Consider the difference $\boldsymbol{q}_{v_{i}}-\boldsymbol{q}_{v_{j}}=N_{G^{\prime}}\left(\boldsymbol{p}_{v_{i}}-\boldsymbol{p}_{v_{j}}\right)$. The factor $N_{G^{\prime}}$ is in the ideal $\alpha\left(v_{i} v_{j}\right)$ by definition of $N_{G^{\prime}}$ and by definition of ideals. Hence the product $N_{G^{\prime}}\left(\boldsymbol{p}_{v_{i}}-\boldsymbol{p}_{v_{j}}\right)$ satisfies the GKM condition for $v_{i}, v_{j}$ in $G$.

The next corollary constructs classes that look like what are called "flow-up" classes in geometric applications. Given a partial order on the vertices of $G$, a flowup class associated to the vertex $v$ is a generalized spline $\boldsymbol{p}^{v}$ so that for each vertex $u$ with $u \ngtr v$ the spline satisfies $\boldsymbol{p}_{u}^{v}=0$. (In geometric applications, flow-up classes satisfy additional conditions as well.) These classes occur naturally in geometric applications: the partial order comes from a suitably generic one-dimensional torus action on the variety (and hence on the graph), and the spline is the cohomology class associated to the subvariety that flows into the vertex $v$. The most famous examples of flow-up classes occur in flag varieties and Grassmannians, where they are known as Schubert classes and where they in fact form a basis for the ring of generalized splines (equivariant cohomology rings, in the geometric context).

Our motivation for the next sequence of corollaries comes from these geometric applications. In those cases, the ideals $\alpha(e)$ for each edge $e$ are principal. If some ideals were not principal, the results that follow could be refined to construct a larger free submodule of $R_{G}$.

We now construct a rank- $n$ free submodule of the generalized splines for an arbitrary edge-labeled graph $(G, \alpha)$ using a collection of linearly independent flowup classes. The reader interested only in the special case of this corollary could prove it directly by taking $G^{\prime}$ to be a single vertex.

Corollary 5.2. Let $R$ be an integral domain and $(G, \alpha)$ a connected edge-labeled graph on $n$ vertices. Then $R_{G}$ contains a free $R$-submodule of rank $n$.

Proof. Enumerate the vertices in $V(G)$ as $v_{1}, v_{2}, \ldots, v_{n}$. For each $v_{i}$ define $G_{i}^{\prime}$ to be the subgraph consisting of exactly vertex $v_{i}$. Clearly $\boldsymbol{p}=\mathbf{1}$ is a generalized spline for $\left(G_{i}^{\prime},\left.\alpha\right|_{G_{i}^{\prime}}\right)$ for all $1 \leq i \leq n$. Then Theorem 5.1 yields generalized splines $\left\{\boldsymbol{q}_{i}: i=1, \ldots, n\right\}$ for $G$, where $\boldsymbol{q}_{i_{v_{j}}}=\delta_{i j} N_{G_{i}^{\prime}}$ and $N_{G_{i}^{\prime}}=\prod_{j \neq i} \alpha_{i, j}$ for arbitrarily chosen $0 \neq \alpha_{i, j} \in \alpha\left(v_{i} v_{j}\right)$. We show that this set is linearly independent in the $R$-module $R_{G}$. Suppose $\sum_{i=1}^{n} c_{i} \boldsymbol{q}_{i}=\mathbf{0}$ for coefficients $c_{i} \in R$. For each $1 \leq j \leq n$, evaluation at $v_{j}$ yields

$$
\sum_{i=1}^{n} c_{i} \boldsymbol{q}_{i_{j}}=\sum_{i=1}^{n} c_{i} \delta_{i j} N_{G_{i}^{\prime}}=c_{j} N_{G_{j}^{\prime}}=0 .
$$


Since $R$ is an integral domain and each $\alpha_{i, j} \neq 0$ it follows that $N_{G_{j}^{\prime}} \neq 0$ for all $j$. Hence (9) implies $c_{j}=0$ for all $1 \leq j \leq n$ so that $\left\{\boldsymbol{q}_{i}: i=1, \ldots, n\right\}$ is linearly independent in $R_{G}$ and therefore spans a free $R$-submodule of rank $n$.

The next corollary makes note of a particular choice for the scaling factor $N_{G^{\prime}}$ in Theorem 5.1 that can be useful in the kinds of examples that arise in geometric applications. All of the hypotheses hold in typical geometric applications (equivariant cohomology with field coefficients, equivariant $K$-theory with field coefficients, and classical algebraic splines).

Corollary 5.3. Fix an edge-labeled graph $(G, \alpha)$ and let $R$ be a unique factorization domain. Suppose that for each edge e the ideal $\alpha(e)$ is principal and choose a generator $\alpha_{i, j}$ for each edge $e=v_{i} v_{j}$. Then for any subgraph $G^{\prime}$ of $G$ we may apply Theorem 5.1 by choosing

$$
N_{G^{\prime}}=\operatorname{lcm}\left\{\alpha_{i, j}: v_{i} v_{j} \in E\left(G-G^{\prime}\right) \text { and } v_{i} \in V\left(G^{\prime}\right) \text { or } v_{j} \in V\left(G^{\prime}\right)\right\} .
$$

The next two corollaries of Theorem 5.1 address particular ways to construct (nontrivial) generalized splines for $G$ from subgraphs of $G$.

Corollary 5.4. If $G$ contains any subgraph $G^{\prime}$ for which $R_{G^{\prime}}$ contains a nontrivial generalized spline then $R_{G}$ also contains a nontrivial generalized spline.

Example 5.5. We can construct generalized splines for the edge-labeled graph $\left(K_{4}, \alpha\right)$ given in Figure 1 using these corollaries. The vertex in the upper-left corner is $v_{1}$ and the other vertices, clockwise around the square, are $v_{2}, v_{3}, v_{4}$. Let $C_{4}$ denote the Hamiltonian cycle determined by ordering the vertices $v_{1} v_{2} v_{3} v_{4}$, and let

$$
N_{C_{4}}=\operatorname{lcm}\left\{\alpha\left(v_{1} v_{3}\right), \alpha\left(v_{2} v_{4}\right)\right\}=\operatorname{lcm}\left\{\alpha_{5}, \alpha_{6}\right\}
$$

with the labeling in Figure 1. Theorem 3.8 constructed many nontrivial generalized splines for $C_{4}$, including

$$
\boldsymbol{p}=\left[\begin{array}{c}
0 \\
\alpha\left(v_{1} v_{4}\right) \alpha\left(v_{1} v_{2}\right) \\
\alpha\left(v_{1} v_{4}\right)\left(\alpha\left(v_{1} v_{2}\right)+\alpha\left(v_{2} v_{3}\right)\right) \\
\alpha\left(v_{1} v_{4}\right)\left(\alpha\left(v_{1} v_{2}\right)+\alpha\left(v_{2} v_{3}\right)+\alpha\left(v_{3} v_{4}\right)\right)
\end{array}\right]=\left[\begin{array}{c}
0 \\
\alpha_{4} \alpha_{1} \\
\alpha_{4}\left(\alpha_{1}+\alpha_{2}\right) \\
\alpha_{4}\left(\alpha_{1}+\alpha_{2}+\alpha_{3}\right)
\end{array}\right] .
$$

The corollaries show that the multiple $N_{C_{4}} \boldsymbol{p}$ is a generalized spline for $K_{4}$.

Corollary 5.6. Let $R$ be an integral domain. If $G$ contains at least two vertices then $R_{G}$ contains a nontrivial generalized spline.

Proof. The vertex set $V$ has at least two vertices, so $V$ has a proper subset. Let $G^{\prime}$ denote a subgraph of $G$ induced by any proper subset of $V$. Choose the unit $\mathbf{1} \in R_{G^{\prime}}$ for the spline $\boldsymbol{p}$ in Theorem 5.1. The factor $N_{G^{\prime}}$ is nonzero because $R$ is an integral domain. 


\section{Decomposing $\boldsymbol{R}_{G}$ as an intersection}

This section describes two ways to express $R_{G}$ as an intersection of rings $R_{G_{i}}$ for simpler graphs $G_{i}$. Both are inspired by the GKM matrix, which allows us to recognize and manipulate the GKM conditions for various subgraphs of $G$.

In the first decomposition, we essentially reorganize the GKM matrix and identify the GKM matrices associated to subgraphs of $G$ inside the GKM matrix for $G$. When these subgraphs are the edges themselves, we recover the result that the generalized splines are the intersection of the GKM conditions on all edges independently. We can alternatively take these subgraphs to be trees, whose generalized splines we identified completely in Section 4; this reduces the number of intersections needed to calculate $R_{G}$.

In the other decomposition, we row-reduce the GKM matrix in a natural way to demonstrate that $R_{G}$ is the intersection of the generalized splines for a particular collection of subcycles of $G$. This demonstrates how the combinatorial perspective can contribute to the study of generalized splines and GKM theory: cycles are subgraphs that do not arise from geometric considerations but are natural in this more general combinatorial setting. It also reinforces Rose's results [1995; 2004] showing the importance of cycles in studying splines. Handschy, Melnick, and Reinders [2014] identify a basis for generalized splines with integer coefficients over cycles in forthcoming work. Bowden, Cao, Hagen, King, and Reinders [2015] give a simpler basis for generalized splines over cycles whose edge labels satisfy a coprimality condition; this allows them to identify the ring structure of the generalized splines completely.

We begin by expressing the ring of generalized splines as an intersection of generalized splines for subgraphs.

Theorem 6.1. Let $(G, \alpha)$ be an edge-labeled graph. Suppose $G_{1}, G_{2}, \ldots, G_{k}$ are a collection of spanning subgraphs of $G$ whose union is $G$, in the sense that $V\left(G_{i}\right)=V(G)$ for all $i$ and $\bigcup_{i=1}^{k} E\left(G_{i}\right)=E(G)$. Let $\alpha_{i}=\left.\alpha\right|_{G_{i}}$ be the edge labelings given by restriction for each $i$. Then

$$
R_{G}=\bigcap_{i=1}^{k} R_{G_{i}} .
$$

Proof. Proposition 2.8 showed that $R_{G}$ is contained in $R_{G^{\prime}}$ for each spanning subgraph $G^{\prime}$ of $G$, and in particular is contained in $R_{G_{i}}$ for each subgraph $G_{i}$. Conversely, suppose $\boldsymbol{p}$ is contained in $\bigcap_{i=1}^{k} R_{G_{i}}$. Every edge $v_{j} v_{k} \in E(G)$ is contained in the edge set of (at least) one of the subgraphs, say $G_{i}$. The spline $\boldsymbol{p}$ is a generalized spline for $G_{i}$ by hypothesis, so the GKM condition is satisfied at $v_{j} v_{k}$ in $G_{i}$ and hence in $G$ as well. 

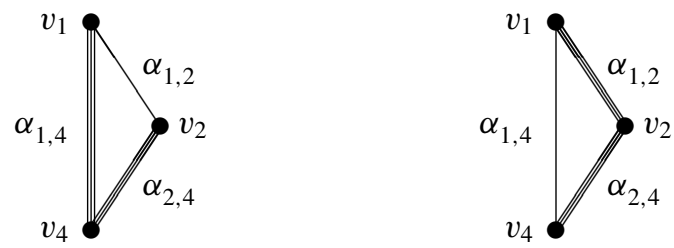

Figure 7. Two spanning trees whose generalized splines determine $R_{C_{3}}$.

Theorem 6.1 generalizes the definition of $R_{G}$. Indeed, for each edge $e \in E(G)$, consider the subgraph $G_{e}=(V(G),\{e\})$. The ring $R_{G_{e}}$ is exactly the subring of $R^{|V(G)|}$ defined by applying the GKM condition at just the edge $e$. Theorem 6.1 says

$$
R_{G}=\bigcap_{e \in E(G)} R_{G_{e}},
$$

namely, that the generalized splines on $G$ are formed by imposing the GKM condition on every edge of $G$ simultaneously.

The next corollary uses another common family of subgraphs: spanning trees. We completely identified the generalized splines for trees in Theorem 4.1. Thus, the corollary expresses the ring of generalized splines using far fewer intersections than in the original GKM formulation. Calculating intersections of subrings is subtle, so this corollary reduces the computational complexity of identifying the ring of generalized splines.

Corollary 6.2. If $G$ can be written as a union of spanning trees $T_{1}, T_{2}, \ldots, T_{m}$ (whose edges are not necessarily disjoint) and if $\alpha_{i}=\left.\alpha\right|_{T_{i}}$ is the edge labeling given by restriction for each $i$ then

$$
R_{G}=\bigcap_{i=1}^{m} R_{T_{i}} .
$$

Figure 7 shows an example using the 3-cycle and principal-ideal edge labels. In this case $R_{G}$ can be expressed as the intersection of just two rings of generalized splines, each of which is completely known. In fact, Theorem 4.1 says that the generalized splines for the two marked paths have the form

$$
\left(p_{1}, p_{1}+\alpha_{1,4} p_{4}+\alpha_{2,4} p_{2}, p_{1}+\alpha_{1,4} p_{4}\right)
$$

and

$$
\left(q_{1}, q_{1}+\alpha_{1,2} q_{2}, q_{1}+\alpha_{1,2} q_{2}+\alpha_{2,4} q_{4}\right)
$$

for free choices of elements $p_{1}, p_{2}, p_{4}, q_{1}, q_{2}, q_{4} \in R$. The intersection of these two sets is $R_{C_{3}}$.

Given a connected graph $G$, we could also use Theorem 6.1 to describe $R_{G}$ in terms of the generalized splines for cycles as follows. Fix a spanning tree $T$ 


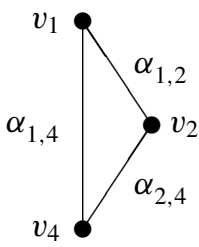

$$
\leftrightarrow\left[\begin{array}{rrr|r}
1 & -1 & 0 & q_{1,2} \alpha_{1,2} \\
0 & 1 & -1 & q_{2,4} \alpha_{2,4} \\
1 & 0 & -1 & q_{1,4} \alpha_{1,4}
\end{array}\right] \leftrightarrow\left[\begin{array}{rrr|l}
1 & -1 & 0 & q_{1,2} \alpha_{1,2} \\
0 & 1 & -1 & q_{2,4} \alpha_{2,4} \\
0 & 0 & 0 & q_{1,4} \alpha_{1,4}-q_{1,2} \alpha_{1,2}-q_{2,4} \alpha_{2,4}
\end{array}\right]
$$

Figure 8. A triangle, its extended GKM matrix, and a row-reduction.

for $G$. For each edge $e \in E(G)-E(T)$ let $C_{e}$ denote the unique cycle contained in $T \cup\{e\}$. (This cycle exists and is unique by a classical result in graph theory [West 2001, pp. 68-69].) Let $C_{e}^{\prime}$ be the graph containing the cycle $C_{e}$ as one connected component and the rest of the vertices of $G$ as the other connected components. Then

$$
R_{G}=R_{T} \cap \bigcap_{e \in E(G)-E(T)} R_{C_{e}^{\prime}}
$$

by Theorem 6.1.

However, a natural row-reduction of the GKM matrix of $G$ proves this intersection directly. To motivate our approach, we return to the complete graph on four vertices with principal-ideal edge labels from Example 3.9. The system of equations in Figure 6 is consistent precisely when $\boldsymbol{q}=\left(q_{1,2}, q_{1,3}, q_{1,4}, q_{2,3}, q_{3,4}\right) \in R^{5}$ satisfies the homogeneous system of equations

$$
\begin{aligned}
& q_{1,2} \alpha_{1,2}-q_{1,4} \alpha_{1,4}+q_{2,4} \alpha_{2,4}=0, \\
& q_{1,3} \alpha_{1,3}-q_{1,4} \alpha_{1,4}+q_{3,4} \alpha_{3,4}=0, \\
& q_{2,3} \alpha_{2,3}-q_{2,4} \alpha_{2,4}+q_{3,4} \alpha_{3,4}=0 .
\end{aligned}
$$

Figure 8 shows the edge-labeled 3 -cycle $v_{1}, v_{2}, v_{4}$ of Figure 7 , its extended GKM matrix, and a natural row-reduction of its extended GKM matrix. The equation that remains is (up to sign) the same as that which occurs in (11). In fact, the entire system in (11) arises from the equations (up to sign) for the three subcycles induced by the vertices

- $v_{1}, v_{2}, v_{4}$,

- $v_{1}, v_{3}, v_{4}$, and

- $v_{2}, v_{3}, v_{4}$. 
The next theorem generalizes this example. We also see it, together with Remark 3.2, as a first step towards generalizing Rose's work on syzygies of edge ideals $[1995 ; 2004]$.

Theorem 6.3. Suppose that $(G, \alpha)$ is an edge-labeled graph on $n$ vertices. Fix a spanning tree $T$ for $G$. For each edge e $\in E(G)-E(T)$ let $C_{e}$ denote the unique cycle contained in $T \cup\{e\}$. Then the extended GKM matrix for $G$ is equivalent to an extended GKM matrix for $T$, followed for each edge $e \in E(G)-E(T)$ by a row that is zero except in the last column, which is $\sum_{e^{\prime} \in C_{e}} q_{e^{\prime}}$ where $q_{e^{\prime}}$ are arbitrary elements of $\alpha\left(e^{\prime}\right)$.

Proof. Choose a spanning tree $T$ for the graph $G$. We assume without loss of generality that the first $n-1$ rows of the GKM matrix for $G$ correspond to the edges in $T$. The first $n-1$ rows of the GKM matrix of $G$ thus consist of the GKM matrix for $T$, by construction.

Consider each of the other rows in turn. Each row corresponds to an edge $e$ in $G$ but not $T$. We now describe an invertible row operation to eliminate all nonzero entries from the first $n$ columns of the row corresponding to $e$ and describe $R_{G}$ more precisely. Denote the edges of the cycle $C_{e}$ by $e_{1}=e=v_{i_{1}} v_{i_{2}}, e_{2}=v_{i_{2}} v_{i_{3}}$, $\ldots, e_{k}=v_{i_{k}} v_{i_{1}}$. Let $c_{j} \in\{ \pm 1\}$ be the entry in the row corresponding to $e_{j}$ and the column corresponding to vertex $v_{i_{j}}$ for each $2 \leq j \neq n$. Denote the $e_{j}$-th row of the GKM matrix by $r_{e_{j}}$. The sum of the scaled rows,

$$
\sum_{j=2}^{k} c_{j} r_{e_{j}},
$$

has 1 in column $v_{i_{2}},-1$ in column $v_{i_{1}}, 0$ in the rest of the first $n$ columns, and $\sum_{j=2}^{k} c_{j} q_{j}$ in the last column, all by the definition of the GKM matrix. Finally we add $\sum_{j=2}^{k} c_{j} r_{e_{j}}$ to the row corresponding to $e$. This leaves 0 in the first $n$ columns of row $e$ and $q_{e}+\sum_{j=2}^{k} c_{j} q_{j}$ in the last entry of the row.

The elements $q_{e}$ and $q_{j}$ are arbitrary elements of their respective ideals and $c_{j}$ is a unit in $R$ for each $j$ so the set of all possible $q_{e}+\sum_{j=2}^{k} c_{j} q_{j}$ is the same as the set of all possible $\sum_{e^{\prime} \in C_{e}} q_{e^{\prime}}$. The result follows.

The last corollary uses this information to describe the generalized splines for $G$ in terms of the generalized splines for cycles, as promised.

Corollary 6.4. Suppose that $(G, \alpha)$ is an edge-labeled graph on $n$ vertices. Fix a spanning tree $T$ for $G$. For each edge e $\in E(G)-E(T)$ let $C_{e}$ denote the unique cycle contained in $T \cup\{e\}$ together with the other vertices in $G$. Then

$$
R_{G}=R_{T} \cap \bigcap_{e \in E(G)-E(T)} R_{C_{e}^{\prime}}
$$


Proof. Consider an edge $e$ outside of the spanning tree $T$ and its corresponding cycle $C_{e}$. The previous theorem showed that the submatrix of an extended GKM matrix for $G$ given by the rows indexed by the edges $e^{\prime} \in E\left(C_{e}\right)$ forms an extended GKM matrix for the cycle $C_{e}$. The vector $\boldsymbol{p} \in R^{|V|}$ solves an extended GKM matrix for $G$ if and only if it simultaneously solves the corresponding extended GKM matrices for $T$ and all of the $C_{e}$ for $e \in E(G)-E(T)$.

\section{Open questions}

We end with several open questions, extending some of the major research problems for splines and GKM theory to the context of generalized splines.

Most research into what we call generalized splines focuses on particular examples, whether because of explicit hypotheses (e.g., a particular choice of the ring $R$, the graph $G$, or the edge-labeling function $\alpha$ ) or implicit hypotheses (e.g., that edge labels be principal). Special cases remain very important, both for applications and for data to build the general theory.

Question 7.1. Identify $R_{G}$ in important special cases: for instance, when all edge labels $\alpha(e)$ are principal ideals; or when $R$ is a particular ring (integers, polynomial rings, ring of Laurent polynomials); or when $G$ is a particular graph or family of graphs (cycles, complete graphs, bipartite graphs, hypercubes).

Splines on complete graphs are particularly important for approximation theory, where they appear as the Alfeld split of a simplex (for a proof see [Tymoczko 2015, Section 3.1]).

Billera asked the following question, seeking an interpretation of $r$-smoothness in the context of equivariant cohomology. We extend Billera's question to ask about the analogue of $r$-smoothness for generalized splines over arbitrary rings.

Question 7.2. Let $(G, \alpha)$ be an edge-labeled graph. Define the function $\alpha^{r}: E \rightarrow \mathcal{I}$ by the condition that for each edge $e$ the image $\alpha^{r}(e)$ is the $r$-th power $(\alpha(e))^{r}$. The $r$-smooth generalized splines are the elements of the ring $R_{G, \alpha^{r}}$. We ask how the $r$-smooth generalized splines compare for various $r$. Billera asks for a geometric interpretation of $r$-smoothness in the context of equivariant cohomology rings.

As a module, the generalized splines $R_{G}$ can also be viewed as group representations: for instance, the group of automorphisms of the graph $G$ that preserve the edge labeling naturally induces a representation on $R_{G}$. Representations obtained in this and similar ways are often intrinsically interesting [Fiebig 2011; Tymoczko 2008] and can also be a powerful tool with which to approach other questions in this section [Tymoczko 2008]. 
Question 7.3. Given a specific automorphism group, what are the induced representations on $R_{G}$ (in terms of irreducible representations, say)? For what families of graphs are there nontrivial representations on $R_{G}$ ?

Propositions 2.8 and 2.10 and Sections 5 and 6 all use combinatorial aspects of graphs to analyze the ring of generalized splines. More recently, Handschy, Melnick, and Reinders [Handschy et al. 2014] and Bowden, Cao, Hagen, King, and Reinders [Bowden et al. 2015] have used deletion and contraction to study splines on cycles. We believe that these are special cases of a more general relationship between the underlying combinatorics and geometry.

Question 7.4. How do classical graph-theoretic constructions (such as deletioncontraction) affect the algebraic structure of splines $R_{G}$ ?

Theorems 2.12, 4.1 and 5.1 are part of a larger program to identify useful bases for splines and GKM modules [Haas 1991; Goldin and Tolman 2009; Guillemin and Zara 2003]. The next question extends that program to generalized splines.

Question 7.5. Given a graph $G$, find a minimal generating set (or basis, if $R$ is an integral domain) for the generalized splines $R_{G}$. If $G$ is a particular family of graphs (cycles, complete graphs, etc.), can we find a minimal generating set (or basis) for $R_{G}$ ?

More specifically, geometers think about bases with certain "upper-triangularity" properties that arise in many important examples, like Schubert classes, BiałynickiBirula classes, and the canonical classes of [Knutson and Tao 2003] and [Goldin and Tolman 2009] (see also [Harada and Tymoczko 2010]). Theorem 5.1 is an initial step in constructing flow-up bases for generalized splines.

Question 7.6. What is the right definition for a flow-up class in the module of generalized splines? Under what conditions is there a flow-up basis for the generalized splines?

Answering the previous question may require further extending generalized splines so that the vertices are labeled by different modules $M_{v}$ rather than a fixed ring $R$, as described in Section 1. Characterizing those splines would have immediate implications in geometric applications like computing equivariant intersection homology.

Question 7.7. Which of the results in this paper extend to generalized splines over modules? Is there an algorithm or an explicit formula to construct flow-up basis classes for generalized spines over modules? 


\section{References}

[Alfeld 1986] P. Alfeld, "On the dimension of multivariate piecewise polynomials", pp. 1-23 in Numerical analysis (Dundee, 1985), edited by D. F. Griffiths and G. A. Watson, Pitman Research Notes in Mathematics Series 140, Longman Scientific and Technical, Harlow, 1986. MR 88d:41014 Zbl 0655.41010

[Alfeld 1987] P. Alfeld, "A case study of multivariate piecewise polynomials", pp. 149-159 in Geometric modeling: algorithms and new trends (Albany, NY, 1985), edited by G. E. Farin, Society for Industrial and Applied Mathematics, Philadelphia, 1987. MR 936451

[Alfeld and Schumaker 1987] P. Alfeld and L. L. Schumaker, "The dimension of bivariate spline spaces of smoothness $r$ for degree $d \geq 4 r+1$ ”, Constr. Approx. 3:2 (1987), 189-197. MR 88e:41025 Zbl 0646.41008

[Alfeld and Schumaker 1990] P. Alfeld and L. L. Schumaker, "On the dimension of bivariate spline spaces of smoothness $r$ and degree $d=3 r+1$ ", Numer. Math. 57:6-7 (1990), 651-661. MR 91h:65022 Zbl 0725.41012

[Alfeld et al. 1987a] P. Alfeld, B. Piper, and L. L. Schumaker, "An explicit basis for $C^{1}$ quartic bivariate splines", SIAM J. Numer. Anal. 24:4 (1987), 891-911. MR 88i:41014 Zbl 0658.65008

[Alfeld et al. 1987b] P. Alfeld, B. Piper, and L. L. Schumaker, "Minimally supported bases for spaces of bivariate piecewise polynomials of smoothness $r$ and degree $d \geq 4 r+1$ ", Comput. Aided Geom. Design 4:1-2 (1987), 105-123. MR 88d:65019 Zbl 0668.41011

[Bahri et al. 2009] A. Bahri, M. Franz, and N. Ray, "The equivariant cohomology ring of weighted projective space", Math. Proc. Cambridge Philos. Soc. 146:2 (2009), 395-405. MR 2010a:57054 Zbl 1205.14022

[Bartels 1984] R. H. Bartels, "Splines in interactive computer graphics", pp. 1-29 in Numerical analysis (Dundee, 1983), edited by D. F. Griffiths, Lecture Notes in Mathematics 1066, Springer, Berlin, 1984. MR 86b:65011 Zbl 0544.65009

[Billera 1988] L. J. Billera, "Homology of smooth splines: generic triangulations and a conjecture of Strang", Trans. Amer. Math. Soc. 310:1 (1988), 325-340. MR 89k:41010 Zbl 0718.41017

[Billera and Rose 1991] L. J. Billera and L. L. Rose, "A dimension series for multivariate splines", Discrete Comput. Geom. 6:2 (1991), 107-128. MR 92g:41010 Zbl 0725.13011

[Billera and Rose 1992] L. J. Billera and L. L. Rose, "Modules of piecewise polynomials and their freeness", Math. Z. 209:4 (1992), 485-497. MR 93f:52017 Zbl 0891.13004

[Bowden and Tymoczko 2015] N. Bowden and J. S. Tymoczko, "Splines mod m", preprint, 2015. arXiv 1501.02027

[Bowden et al. 2015] N. Bowden, S. Hagen, M. King, and S. Reinders, "Bases and structure constants of generalized splines with integer coefficients on cycles", preprint, 2015. arXiv 1502.00176

[Braden and MacPherson 2001] T. Braden and R. MacPherson, "From moment graphs to intersection cohomology”, Math. Ann. 321:3 (2001), 533-551. MR 2003g:14030 Zbl 1077.14522

[Brion 1996] M. Brion, "Piecewise polynomial functions, convex polytopes and enumerative geometry", pp. 25-44 in Parameter spaces (Warsaw, 1994), edited by P. Pragacz, Banach Center Publications 36, Polish Academy of Science, Warsaw, 1996. MR 99h:14052 Zbl 0878.14035

[Chang and Skjelbred 1974] T. Chang and T. Skjelbred, "The topological Schur lemma and related results", Ann. of Math. (2) 100 (1974), 307-321. MR 51 \#11552 Zbl 0249.57023

[Chui and He 1989] C. K. Chui and T. X. He, "On the dimension of bivariate superspline spaces", Math. Comp. 53:187 (1989), 219-234. MR 90c:41014 Zbl 0668.41013 
[Chui and Lai 1985] C. K. Chui and M. J. Lai, "On bivariate vertex splines", pp. 84-115 in Multivariate approximation theory, III (Oberwolfach, 1985), edited by W. Schempp and K. Zeller, Internationale Schriftenreihe zur Numerischen Mathematik 75, Birkhäuser, Basel, 1985. MR 88m:41002 Zbl 0588.65009

[Chui and Lai 1990] C. K. Chui and M. J. Lai, "Multivariate vertex splines and finite elements", J. Approx. Theory 60:3 (1990), 245-343. MR 91e:65022 Zbl 0702.41017

[Dalbec and Schenck 2001] J. P. Dalbec and H. K. Schenck, "On a conjecture of Rose", J. Pure Appl. Algebra 165:2 (2001), 151-154. MR 2002i:41046 Zbl 1089.41029

[DiPasquale 2012] M. R. DiPasquale, "Shellability and freeness of continuous splines", J. Pure Appl. Algebra 216:11 (2012), 2519-2523. MR 2927183 Zbl 1300.13012

[Fiebig 2011] P. Fiebig, "Sheaves on affine Schubert varieties, modular representations, and Lusztig's conjecture", J. Amer. Math. Soc. 24:1 (2011), 133-181. MR 2012a:20072 Zbl 1270.20053

[Gmelig Meyling and Pfluger 1985] R. H. J. Gmelig Meyling and P. R. Pfluger, "On the dimension of the spline space $S_{2}^{1}(\Delta)$ in special cases", pp. 180-190 in Multivariate approximation theory, III (Oberwolfach, 1985), edited by W. Schempp and K. Zeller, Internationale Schriftenreihe zur Numerischen Mathematik 75, Birkhäuser, Basel, 1985. MR 88f:65024 Zbl 0567.41015

[Goldin and Tolman 2009] R. F. Goldin and S. Tolman, "Towards generalizing Schubert calculus in the symplectic category”, J. Symplectic Geom. 7:4 (2009), 449-473. MR 2010k:53143 Zbl 1185.57024

[Goresky et al. 1998] M. Goresky, R. Kottwitz, and R. MacPherson, "Equivariant cohomology, Koszul duality, and the localization theorem”, Invent. Math. 131:1 (1998), 25-83. MR 99c:55009 Zbl 0897.22009

[Guillemin and Zara 2001] V. Guillemin and C. Zara, "1-skeleta, Betti numbers, and equivariant cohomology”, Duke Math. J. 107:2 (2001), 283-349. MR 2002j:53110 Zbl 1020.57013

[Guillemin and Zara 2003] V. Guillemin and C. Zara, "The existence of generating families for the cohomology ring of a graph”, Adv. Math. 174:1 (2003), 115-153. MR 2004f:53099 Zbl 1020.57014

[Guillemin et al. 2006] V. Guillemin, T. S. Holm, and C. Zara, "A GKM description of the equivariant cohomology ring of a homogeneous space", J. Algebraic Combin. 23:1 (2006), 21-41. MR 2008b:55010 Zbl 1096.53050

[Haas 1991] R. Haas, "Module and vector space bases for spline spaces", J. Approx. Theory 65:1 (1991), 73-89. MR 92b:41021 Zbl 0732.41014

[Handschy et al. 2014] M. Handschy, J. Melnick, and S. Reinders, "Integer generalized splines on cycles", preprint, 2014. arXiv 1409.1481

[Harada and Tymoczko 2010] M. Harada and J. S. Tymoczko, "Poset pinball, GKM-compatible subspaces, and Hessenberg varieties", preprint, 2010. arXiv 1007.2750

[Harada et al. 2005] M. Harada, A. Henriques, and T. S. Holm, "Computation of generalized equivariant cohomologies of Kac-Moody flag varieties”, Adv. Math. 197:1 (2005), 198-221. MR 2006h:53086 Zbl 1110.55003

[Holm 2008] T. S. Holm, "Act globally, compute locally: group actions, fixed points, and localization", pp. 179-195 in Toric topology, edited by M. Harada et al., Contemporary Mathematics 460, American Mathematical Society, Providence, RI, 2008. MR 2009h:53191 Zbl 1155.53051

[Knutson and Tao 2003] A. Knutson and T. Tao, "Puzzles and (equivariant) cohomology of Grassmannians”, Duke Math. J. 119:2 (2003), 221-260. MR 2006a:14088 Zbl 1064.14063

[McDonald 2007] T. McDonald, "Splines with boundary conditions", Comput. Math. Appl. 54:9-10 (2007), 1234-1239. MR 2009b:65021 Zbl 1140.41001 
[McDonald and Schenck 2009] T. McDonald and H. K. Schenck, "Piecewise polynomials on polyhedral complexes", Adv. in Appl. Math. 42:1 (2009), 82-93. MR 2009k:41013 Zbl 1178.41008

[Morgan and Scott 1975] J. Morgan and R. Scott, "A nodal basis for $C^{1}$ piecewise polynomials of degree $n \geq$ 5", Math. Comp. 29:131 (1975), 736-740. MR 51 \#11930 Zbl 0307.65074

[Mourrain and Villamizar 2013] B. Mourrain and N. Villamizar, "Homological techniques for the analysis of the dimension of triangular spline spaces", J. Symbolic Comput. 50 (2013), 564-577. MR 2996896 Zbl 06124247

[Payne 2006] S. Payne, "Equivariant Chow cohomology of toric varieties", Math. Res. Lett. 13:1 (2006), 29-41. MR 2007f:14052 Zbl 1094.14036

[Rose 1995] L. L. Rose, "Combinatorial and topological invariants of modules of piecewise polynomials”, Adv. Math. 116:1 (1995), 34-45. MR 97b:13036 Zbl 0837.57020

[Rose 2004] L. L. Rose, "Graphs, syzygies, and multivariate splines", Discrete Comput. Geom. 32:4 (2004), 623-637. MR 2005g:41024 Zbl 1069.41012

[Schenck 1997] H. K. Schenck, Homological methods in the theory of splines, thesis, Cornell University, Ithaca, NY, 1997, available at http://search.proquest.com/docview/304343601. MR 2695686

[Schenck 2012] H. K. Schenck, "Equivariant Chow cohomology of nonsimplicial toric varieties", Trans. Amer. Math. Soc. 364:8 (2012), 4041-4051. MR 2912444 Zbl 06191303

[Schenck and Stillman 1997] H. K. Schenck and M. Stillman, "Local cohomology of bivariate splines”, J. Pure Appl. Algebra 117/118 (1997), 535-548. MR 99d:13011 Zbl 0902.41010

[Schumaker 1979] L. L. Schumaker, "Lower bounds for spline approximation”, pp. 213-223 in Approximation theory: VIth semester, Stefan Banach International Mathematics Center (Warsaw, 1975), edited by Z. Ciesielski, Banach Center Publications 4, Polish Academy of Science, Warsaw, 1979. MR 81h:41011 Zbl 0418.41005

[Schumaker 1984a] L. L. Schumaker, "Bounds on the dimension of spaces of multivariate piecewise polynomials", Rocky Mountain J. Math. 14:1 (1984), 251-264. MR 85h:41091 Zbl 0601.41034

[Schumaker 1984b] L. L. Schumaker, "On spaces of piecewise polynomials in two variables", pp. 151-197 in Approximation theory and spline functions (St. John's, Newfoundland, 1983), edited by S. P. Singh et al., NATO Advanced Science Institute Series C Mathematical and Physical Sciences 136, Reidel, Dordrecht, 1984. MR 86j:41009 Zbl 0553.41018

[Schumaker 1988] L. L. Schumaker, "Dual bases for spline spaces on cells", Comput. Aided Geom. Design 5:4 (1988), 277-284. MR 90a:41013 Zbl 0652.41012

[Tymoczko 2005] J. S. Tymoczko, "An introduction to equivariant cohomology and homology, following Goresky, Kottwitz, and MacPherson", pp. 169-188 in Snowbird lectures in algebraic geometry, edited by R. Vakil, Contemporary Mathematics 388, American Mathematical Society, Providence, RI, 2005. MR 2006m:55019

[Tymoczko 2008] J. S. Tymoczko, "Permutation actions on equivariant cohomology of flag varieties", pp. 365-384 in Toric topology, edited by M. Harada et al., Contemporary Mathematics 460, American Mathematical Society, Providence, RI, 2008. MR 2010i:14090 Zbl 1147.14024

[Tymoczko 2015] J. S. Tymoczko, "Splines in geometry and topology", Comput. Aided Geom. Design (online publication November 2015).

[Wang 2000] R.-H. Wang, "Multivariate spline and algebraic geometry", J. Comput. Appl. Math. 121:1-2 (2000), 153-163. MR 1780047 Zbl 0960.41008

[West 2001] D. B. West, Introduction to graph theory, 2nd ed., Prentice Hall, Upper Saddle River, NJ, 2001. MR 96i:05001 Zbl 0845.05001 
[Yuzvinsky 1992] S. Yuzvinsky, "Modules of splines on polyhedral complexes", Math. Z. 210:2 (1992), 245-254. MR 93h:52015 Zbl 0848.52003

[Zhu and Wang 2005] C.-G. Zhu and R.-H. Wang, "Piecewise semialgebraic sets", J. Comput. Math. 23:5 (2005), 503-512. MR 2006d:14071 Zbl 1084.14058

[Zhu and Wang 2011] C.-G. Zhu and R.-H. Wang, "The correspondence between multivariate spline ideals and piecewise algebraic varieties”, J. Comput. Appl. Math. 236:5 (2011), 793-800. MR 2853504 Zbl 1236.65012

Received March 4, 2015. Revised August 20, 2015.

\section{SIMCHA GILBERT}

Department of Mathematics and Statistics

SMITH COLLEGE

44 College Lane

NORTHAMPTON, MA 01063

UNITED STATES

sgilbert@smith.edu

JULIANNA TYMOCZKO

DEPARTMENT OF MATHEMATICS AND STATISTICS

SMith College

44 College Lane

NORTHAMPTON, MA 01063

UNITED STATES

jtymoczko@smith.edu

SHIRA VIEL

DEPARTMENT OF MATHEMATICS

NorTh CAROLINA STATE UNIVERSITY

2108 SAS HALL

RALEIGH, NC 27695

UNITED STATES

spolste@ncsu.edu 


\title{
PACIFIC JOURNAL OF MATHEMATICS
}

\author{
msp.org/pjm
}

Founded in 1951 by E. F. Beckenbach (1906-1982) and F. Wolf (1904-1989)

\section{EDITORS}

Don Blasius (Managing Editor)

Department of Mathematics

University of California

Los Angeles, CA 90095-1555

blasius@math.ucla.edu

\author{
Paul Balmer \\ Department of Mathematics \\ University of California \\ Los Angeles, CA 90095-1555 \\ balmer@math.ucla.edu \\ Robert Finn \\ Department of Mathematics \\ Stanford University \\ Stanford, CA 94305-2125 \\ finn@math.stanford.edu \\ Sorin Popa \\ Department of Mathematics \\ University of California \\ Los Angeles, CA 90095-1555 \\ popa@math.ucla.edu
}

\author{
Vyjayanthi Chari \\ Department of Mathematics \\ University of California \\ Riverside, CA 92521-0135 \\ chari@math.ucr.edu \\ Kefeng Liu \\ Department of Mathematics \\ University of California \\ Los Angeles, CA 90095-1555 \\ liu@math.ucla.edu \\ Jie Qing \\ Department of Mathematics \\ University of California \\ Santa Cruz, CA 95064 \\ qing@ cats.ucsc.edu
}

\section{PRODUCTION}

Silvio Levy, Scientific Editor, production@msp.org

\section{SUPPORTING INSTITUTIONS}

ACADEMIA SINICA, TAIPEI

CALIFORNIA INST. OF TECHNOLOGY

INST. DE MATEMÁTICA PURA E APLICADA

KEIO UNIVERSITY

MATH. SCIENCES RESEARCH INSTITUTE

NEW MEXICO STATE UNIV.

OREGON STATE UNIV.

\author{
STANFORD UNIVERSITY \\ UNIV. OF BRITISH COLUMBIA \\ UNIV. OF CALIFORNIA, BERKELEY \\ UNIV. OF CALIFORNIA, DAVIS \\ UNIV. OF CALIFORNIA, LOS ANGELES \\ UNIV. OF CALIFORNIA, RIVERSIDE \\ UNIV. OF CALIFORNIA, SAN DIEGO \\ UNIV. OF CALIF., SANTA BARBARA
}

\author{
Daryl Cooper \\ Department of Mathematics \\ University of California \\ Santa Barbara, CA 93106-3080 \\ cooper@math.ucsb.edu \\ Jiang-Hua Lu \\ Department of Mathematics \\ The University of Hong Kong \\ Pokfulam Rd., Hong Kong \\ jhlu@maths.hku.hk \\ Paul Yang \\ Department of Mathematics \\ Princeton University \\ Princeton NJ 08544-1000 \\ yang@math.princeton.edu
}

These supporting institutions contribute to the cost of publication of this Journal, but they are not owners or publishers and have no responsibility for its contents or policies.

See inside back cover or msp.org/pjm for submission instructions.

The subscription price for 2016 is US $\$ 440 /$ year for the electronic version, and $\$ 600 /$ year for print and electronic.

Subscriptions, requests for back issues and changes of subscribers address should be sent to Pacific Journal of Mathematics, P.O. Box 4163, Berkeley, CA 94704-0163, U.S.A. The Pacific Journal of Mathematics is indexed by Mathematical Reviews, Zentralblatt MATH, PASCAL CNRS Index, Referativnyi Zhurnal, Current Mathematical Publications and Web of Knowledge (Science Citation Index).

The Pacific Journal of Mathematics (ISSN 0030-8730) at the University of California, c/o Department of Mathematics, 798 Evans Hall \#3840, Berkeley, CA 94720-3840, is published twelve times a year. Periodical rate postage paid at Berkeley, CA 94704, and additional mailing offices. POSTMASTER: send address changes to Pacific Journal of Mathematics, P.O. Box 4163, Berkeley, CA 94704-0163.

PJM peer review and production are managed by EditFLOW ${ }^{\circledR}$ from Mathematical Sciences Publishers.

\section{PUBLISHED BY}

\section{mathematical sciences publishers \\ nonprofit scientific publishing}

http://msp.org/

(C) 2016 Mathematical Sciences Publishers 


\title{
PACIFIC JOURNAL OF MATHEMATICS
}

\author{
Volume $281 \quad$ No. $2 \quad$ April 2016
}

The Eisenstein elements of modular symbols for level product of two 257 distinct odd primes

DEBARGHA BANERJEE and SRILAKSHMI KRISHNAMOORTHY

Primitively generated Hall algebras

ARKADY BERENSTEIN and JACOB GREENSTEIN

Generalized splines on arbitrary graphs

Simcha Gilbert, JULiAnNA TyMOCZKO and SHIRA ViEL

Good traces for not necessarily simple dimension groups

DAVID HANDELMAN

On Fourier coefficients of certain residual representations of symplectic groups

DIHUA JIANG and BAIYING LIU

On the existence of central fans of capillary surfaces

AMMAR KHANFER

Surfaces of prescribed mean curvature $H(x, y, z)$ with one-to-one central projection onto a plane

FRIEDRICH SAUVIGNY 